274 L. Wenger, Der Eid in den griechischen Papyrusurkunden.

Gold zahlen. Dies wird wohl, wenn auch die nachfolgenden Buchstaben $\mu \varepsilon \varepsilon \tau o($ ?) keinen mir verständlichen Sinn ergeben, der Satz.zu bedeuten haben. Der copulativ angeschlossene Satz aber bedeutet doch nur die tautologische Bestimmung, dass im Falle der Nichterfüllung seiner Verpflichtung der Aussteller der Gestellungsurkunde pro capite der nicht gestellten Personen der genannten Geldstrafe unterliegen solle. So versucht man es in verschiedener Weise die Erfüllung: einer eidlich übernommenen Verbindlichkeit zu sichern; wie wenig man dem Bestärkungsmittel des Eides selbst Bedeutung beimafs, lässt sich aber hieraus indirect entnehmen.

\title{
$\mathrm{X}$. \\ Romanistische Papyrusstudien.
}

Von

\section{Ludwig Mitteis.}

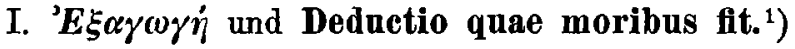

Pap. Grenfell I Nr. $11^{2}$ ) vom Jahr 157 v. Chr. lautet folgendermalsen:

Col. I.

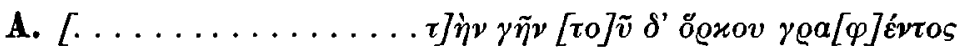

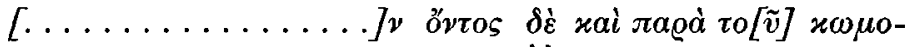

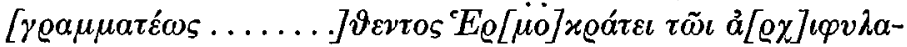

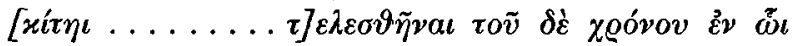

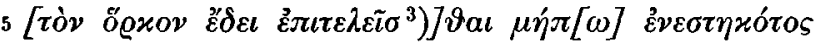

1) Nach Fertigstellung dieser Abhandlung erschien das erste Heft des zweiten Bandes rom Arch. f. Papyrusforschung, in welchem Naber (p. 38-40) einige kurze, aber sachlich zutreffende Bemerkungen zur Prozessgeschichte unseres Papyrus und Conjecturen zur Lesung liefert. $\mathrm{Zu}$ einer Abänderung des von mir Gesagten sah ich mich nirgends veranlasst und auf die hier vor Allem interessirende Frage der $\xi \xi \alpha \gamma \omega \gamma \eta$ ist $N$. überhaupt nicht eingetreten; doch habe ich auf seine sachlichen Erläuterungen gelegentlich noch Rücksicht nehmen können und vor Allem seine und Wilamowitz' Vorschläge zur Lesung in den Abdruck der Urkunde aufgenommen. - ${ }^{2}$ ) Grenfell, an Alexandrian erotic fragment and other Greek Papyri Oxford 1896 p. 25 ff. -- 3) Ergänzt von Naber. 


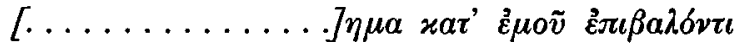

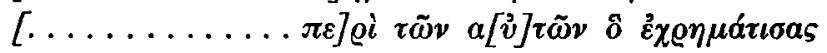

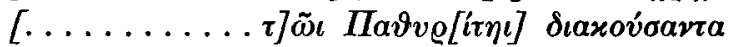

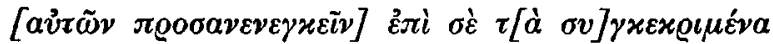

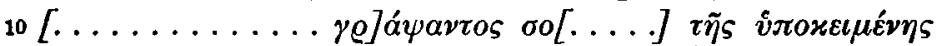

$[\ldots \ldots \ldots$. . . .

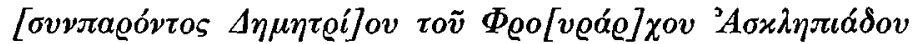

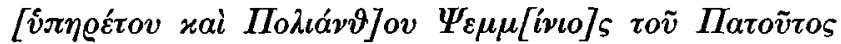

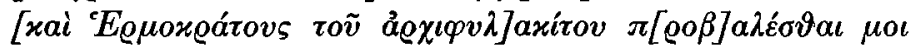
toùs $\pi \varepsilon \varrho i$

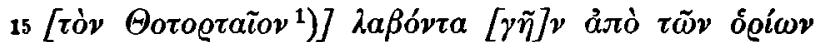

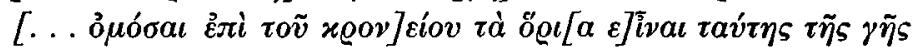

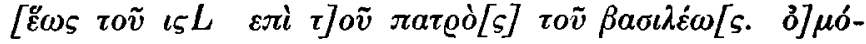

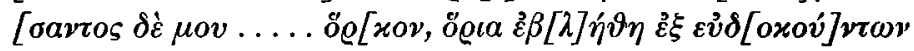

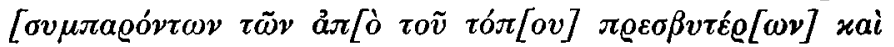

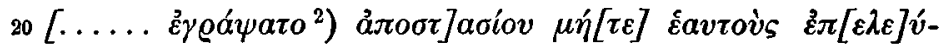
$\sigma \varepsilon \sigma \vartheta$

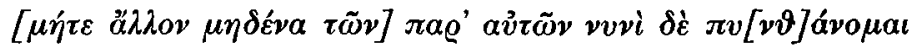

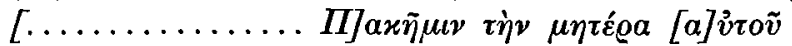

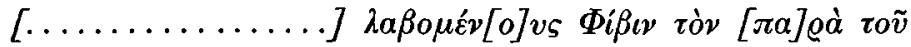

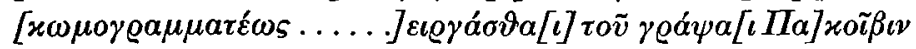
$25 \ldots \ldots \ldots \ldots \ldots \ldots \ldots \ldots.] \varepsilon \sigma \vartheta \omega \sigma[.] \tau \tilde{\omega} \iota$ $\varkappa \omega \mu[о \gamma \varrho \alpha \mu] \mu \alpha \tau \varepsilon \tilde{\imath}$ $\vec{\varepsilon} \mu \tilde{\eta} \varsigma$

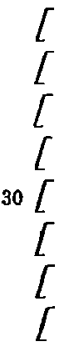

$$
\begin{aligned}
& \text { ]evros } x[\ldots]
\end{aligned}
$$

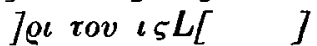

$$
\begin{aligned}
& \text { ]x@óvov } \tau \alpha[\ldots \ldots]\rceil \eta \\
& 7 \mu o \text { ла } \\
& ] \tau \tilde{\eta} s \text { ov } \nu[ \\
& \text { ]xótos xai } \varepsilon[ \\
& ] \not{a} \xi \iota \tilde{\omega} \quad \sigma \dot{\varepsilon} \quad \gamma \varrho[a ́ \psi \alpha \iota \\
& \text { Col. II. } \\
& ] \pi \varepsilon \iota \nu \vec{\varepsilon} \pi \iota[
\end{aligned}
$$

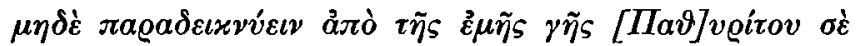

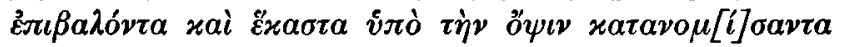

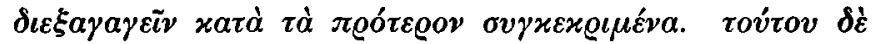

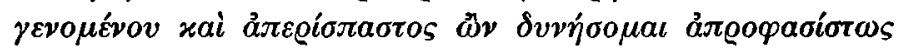

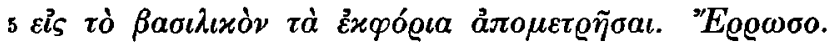

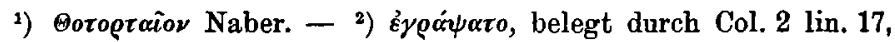
kann der Grenfell'schen Ergänzung unbedingt hinzugefügt werden. 


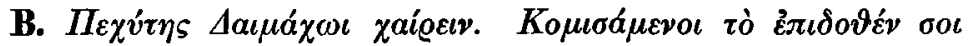

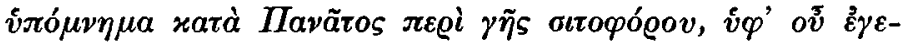

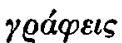

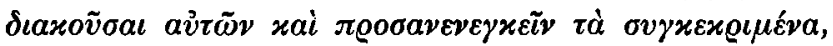

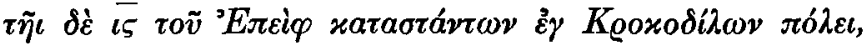

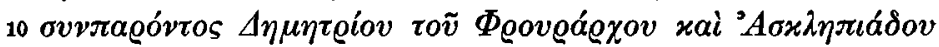

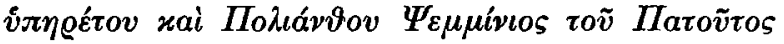

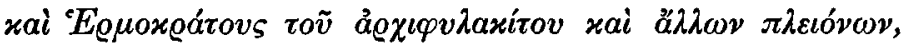

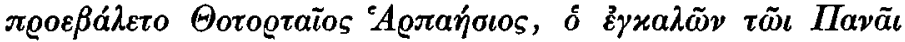
$\pi \varepsilon \underline{i} i \tilde{\eta} s$

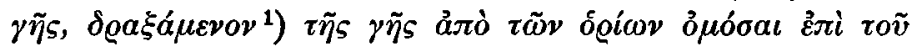

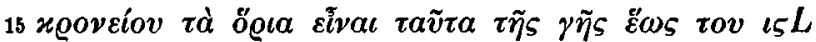

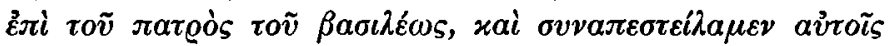

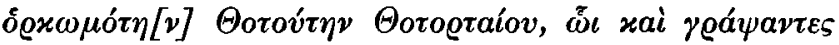

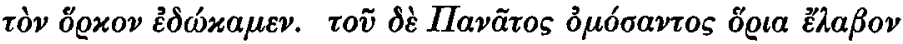

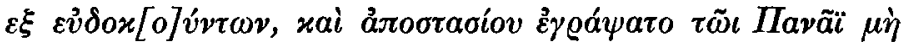
$\dot{\varepsilon} \pi \varepsilon \lambda \varepsilon \dot{v}-$

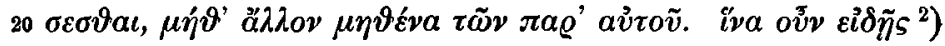

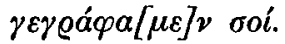

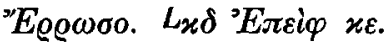

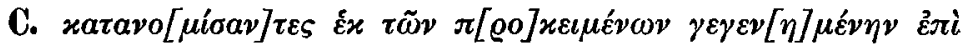

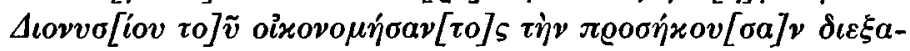
$\gamma \omega \gamma \dot{\eta} \boldsymbol{\nu}$

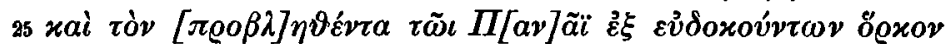

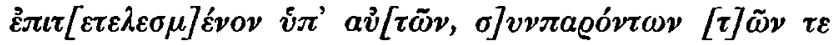

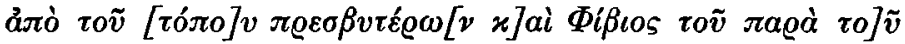
$x \omega \mu \mathrm{\gamma}$

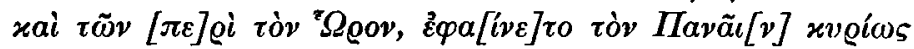
$\ddot{\varepsilon} \chi \varepsilon \varepsilon \nu$

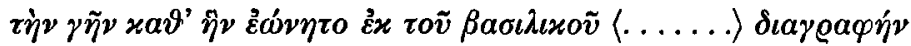
30

〈'Ev่vช์ $\chi \varepsilon \iota\rangle$

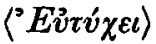

Dieser bereits im J. 1896 von Grenfell veröffentlichte Papyrus beansprucht ein bedeutendes rechtshistorisches Interesse und ich hole gern das von mir und Andern bisher Versäumte nach, indem ich auf seinen merkwürdigen Inhalt

1) Bei Grenfell $\delta \varrho v \xi \alpha \dot{\alpha \varepsilon \nu \nu \nu . ~ S . ~ u n t e n ~ S . ~} 283$ N. 1. - ${ }^{2}$ ) Grenfell schreibt $\varepsilon \gamma \vartheta \tilde{\eta} s$; die Correctur $\varepsilon i d \tilde{\eta} s$ stammt von Naber. 
hinweise. Die Erörterung desselben stösst zwar auf einige Schwierigkeit wegen des üblen Zustands, in welchem uns wenigstens die erste Hälfte überliefert ist; aber das für die Rechtsgeschichte Wesentliche kann, glaube ich, aus dem fast vollständig erhaltenen zweiten Theil mit aller Sicherheit entnommen werden.

Der in dem Stück enthaltene Urkundencomplex zerfällt deutlich in drei von einander wohl zu sondernde Theile, von denen der erste (A) nur lückenhaft erhaltene die ganze Col. I und von Col. II die ersten fünf Zeilen umfasst. Der zweite Theil (B) reicht von Col. II 6 bis 22, der dritte (C) bis zum Schluss. Das Präscript des ersten Theils, welches die scharfe Bestimmung der Sachlage wesentlich erleichtert haben würde, ist leider verloren gegangen.

In der Auffassung des zwischen den drei Gliedern des Papyrus obwaltenden Verhältnisses weiche ich vom Herausgeber $a b$; die schlechte Erhaltung des Stückes bedingt es eben, dass das Verständniss leicht auseinandergeht. Ich will zur Vereinfachung zunächst meine Anschauung über den Zusammenhang der drei Theile vorausschicken, um von dieser Grundlage aus mich mit Grenfell's Auslegung auseinanderzusetzen.

Es handelt sich um einen Eigenthumsstreit betreffend eine im Pathyritischen Gau zwischen den Grundstücken zweier Besitzer, Panas und Thotortaios belegene Parzelle; dass das Eigenthum und nicht bloss der Besitz in Frage steht, ergibt sich aus der Urtheilssentenz Col. II lin. 28:

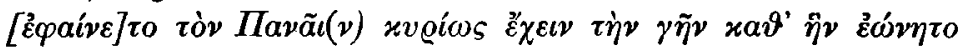

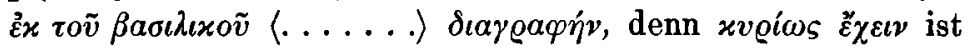

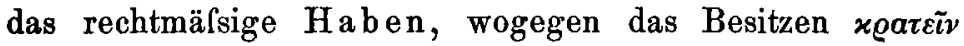
genannt wird ${ }^{1}$, und ausserdem ist ja sogar auf den Rechtstitel des Eigenthümers Bezug genommen, nämlich auf den

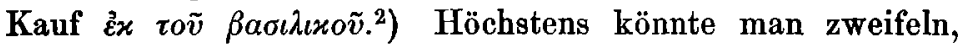
ob das anerkannte Recht des Panas wahres Eigenthum oder

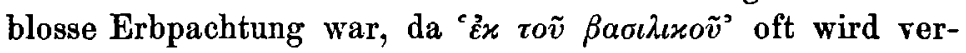
erbpachtet worden sein; doch wissen wir, dass die könig-

1) Vgl. meine Bem. Arch. f. Pap.-Forsch. 1, 188 n. 1. Belege sind überflüssig. $-{ }^{2}$ ) D. h. vom Domanialfiscus. 
liche Domäne in Aegypten gar nicht selten auch Landverkäufe vorgenommen hat, namentlich wo es sich um confiscirte Güter handelt ${ }^{1}$ ), und kann jedenfalls für die hier in Rede stehende Frage ein Fehler nicht entstehn, wenn wir einen wahren Eigenthumsstreit voraussetzen. ${ }^{2}$ )

a) Von den drei Haupttheilen, in welche der Papyrus sich gliedert, führen nun die beiden letzten Col. II 6-30 jedenfalls auf ältere Vorgänge zurück, als der Eingang ist. 'Pechytes', so lautet es in Col. II 6-22, 'grüsst den Daimachos. Nachdem wir das Gesuch empfangen, das Dir überreicht worden war gegen Panas wegen eines Getreidefelds, worauf Du den Bescheid geschrieben hattest, sie (d. h. die Kläger) zu hören und das Erkenntniss vorzulegen, und als sodann am $16^{\text {ten }}$ Epeiph Termin abgehalten worden war in Krokodilopolis im Beisein des Demetrios des Phrurarchen und des Officials Asklepiades und des Polianthos und Psemminios des Sohnes des Patus und des Archiphylakiten und anderer mehr, machte Thotortaios der Sohn des Harpaësis, der den Panas wegen des Feldes belangt, den Vorschlag, jener solle sich des Feldes bemächtigen von den Grenzen und dann beim Kronostempel schwören, dass dieses die Grenzen des Feldes sind bis zum $16^{\text {ten }}$ Jahr des Vaters des Königs, und wir gaben ihnen mit als Eidesempfänger den Thotutes Sohn des Thotortaios, dem wir auch den schriftlichen Entwurf des Eides einhändigten. Da nun Panas den Eid leistete, zog ich die Grenzen in ihrem Einverständniss und es wurde eine Abstandsurkunde geschrieben, dem Panas nicht hinzuzugehn, auch solle Niemand andrer von seiner Seite das thun. Damit Du es nun wissest, haben wir Dir geschrieben. Leb wohl. Im $24^{\text {ten }} \mathrm{Jahr}^{3}$ ) am $25^{\text {ten }}$ Epeiph.

1) Beispiele geben die von Wilcken herausgegebenen Acten der Bank von Theben (Abh. d. preuss. Akad. 1886) oder CPR. 1 Nr. 1 lin. 5 oder P. Amh. 1 n. 97, letztere beide aus der römischen Zeit. - BGU. 18

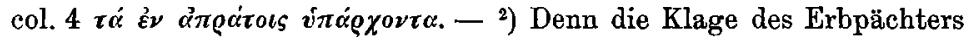
gegen dritte Prätendenten ist ersichtlich die dingliche und muss sich bei den Ptolemäern ebenso in den Formen der Vindication bewegt haben wie die römische Klage 'si ager vectigalis petetur'. - ${ }^{3}$ ) Wahrscheinlich ist das 24. Jahr des Ptolemaios Philometor gemeint; Grenfell Note 22. 
Obwohl hiervon manches schwierig ist und erst späterer Erläuterung bedarf, ist doch soviel klar, dass Pechytes hier ein Unterbeamter ist, der seinem Vorgesetzten Daimachos über den Vollzug eines Auftrags berichtet, und es ist daher sicher unhaltbar, wenn die Erläuterung des Herausgebers (p. 25) den Daimachus bezeichnet als 'a subordinate official', welcher an 'Pechutes a higher official' berichtet; das Verhältniss ist gerade das Umgekehrte. Das geht aus dem Umstand unbestreitbar hervor, dass Daimachos das Gesuch

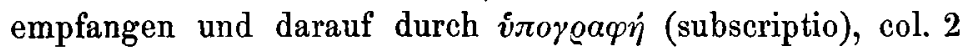
lin. 7 Bescheid gesetzt hatte, die Parteien zu vernehmen; Pechytes fungirt dabei also als commissarischer Richter; wieweit der Auftrag ging, ob bloss auf Beweisaufnahme ${ }^{1}$ ) oder auf ein Schlusserkenntniss, mag vor der Hand offen bleiben. Dass Daimachos der Vorgesetzte ist, kann angesichts dieses zwingenden sachlichen Grundes nicht deswegen bezweifelt werden, weil Pechytes ihn ohne jede Devotionsformel apostrophirt, denn es wäre erst zu zeigen, dass solche im ptolemäischen Dienst (ausser gegenüber dem König) nothwendig

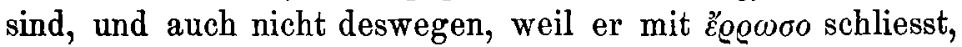
statt des gegen Höhere sonst gebrauchten $\varepsilon v ̉ \tau v ́ \chi \varepsilon \iota$; denn dieselbe cordiale Schlussformel gebraucht in unserm Papyrus auch die Privatpartei.

b) Das Anfangsstück, Col. I und Col. II, 1-6 liegt, was auch Grenfell voraussetzt, jedenfalls zeitlich hinter der eben besprochenen Amtshandlung des Pechytes. Das geht daraus hervor, dass der darin geschilderte Vorgang, wie wir trotz der mangelhaften Erhaltung sehen können, in Allem, namentlich auch in Bezug auf die daran betheiligten Nebenpersonen mit dem im zweiten Abschnitt referirten genau übereinstimmt ${ }^{2}$ ) und daher offenbar eine zweite Erzählung dieses Vorgangs vorliegt. Es ist danach nicht möglich an der Identität des Thatbestandes in beiden Referaten zu zweifeln

1) Vgl. Hermes 30, 581. - ${ }^{2}$ ) Die Namen sind zwar in diesem Stück theilweise lädirt, aber doch mit Sicherheit zu ergänzen; der einzige Zweifel kann bezüglich des Polianthos (col. 1 lin. 13) bleiben,

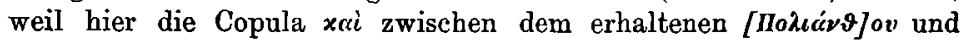
dem darauffolgenden $I I \sigma \varepsilon \mu \mu[i \nu l]$ os abgeht. Aber wahrscheinlich liegt ein blosses Omissum vor. 
und etwa anzunehmen, dass von zwei verschiedenen Tagfahrten über die Grenzfeststellung die Rede sei.

Seinem Inhalt nach ist dieser einleitende Theil eine Beschwerde darüber, dass trotz dem geleisteten Eid der Gegner die Grenze neuerlich nicht respectirt; es ist klar, dass diese Beschwerde, obwohl der Name ihres Verfassers mit dem Präscript verloren gegangen ist, von Panas herrühren muss. Auch ist leicht zu ersehen, dass dieser Theil der eigentliche Träger der ganzen Schrift ist, an welchen sich die beiden anderen nur in einer noch näher zu bestimmenden Function anschliessen.

c) Der Schluss, Col. II bei 23-29 lautet wie folgt: 'Indem wir aus dem Vorliegenden erkennen, dass die gebührende Entscheidung bei Dionysios dem gewesenen Oekonomen erfolgt, und der dem Panas einverständlich (auferlegte) Eid von ihm [abgelegt worden ist], wobei die Aeltesten des Orts und Phibis der Dorfschreibergehilfe und die Genossen des Horos zugegen waren, - ist es erhellt, dass Panas das Land zu Recht hat, gemäls seiner Kaufsurkunde von der Domäne?.

Das Verhältniss dieses Theils zu den beiden andern möchte ich weder so bestimmen, wie es der Herausgeber, noch auch, wie es - davon abweichend - neuestens Naber thut. Grenfell meint, es liege hier die Antwort des (übrigens nicht genannten) Richters auf die unter b) bezeichnete Eingabe vor. Aber erstens kommt es $\mathrm{m}$. W. nie vor, dass das Urtheil auf den Körper des Klaglibells hinaufgeschrieben wird, sondern dasselbe bildet äusserlich eine selbständige Urkunde; ausserdem scheint nach Grenfell's Angabe der Papyrus von einer einzigen Hand geschrieben zu sein (written in a large clear official hand), während man bei seiner Annahme einen Wechsel der Schriftzüge erwartet. Und könnte man sich auch über diese äusseren Bedenken hinwegsetzen, so steht immer noch die Formulirung dieses Theils entgegen; denn wie sagt unser Schlusssatz? 'Erkennend auf Grund des Vorstehenden, dass ....' - das wäre ja ganz unmöglich, das neue Urtheil wäre ja nicht gefällt worden auf Grund des „Vorstehenden" allein, d. h. der Eingabe Col. 1 - Col. 2, 6 oder der in der Vergangenheit liegenden Verhandlung Col. 2, $6 \mathrm{f}$, 
sondern hätte eine neue Verhandlung zur Grundlage haben müssen. Freilich konnte nach Grenfell's Annahme Panas sich schon auf ein früheres Urtheil stützen, und dessen Vollstreckung verlangen; dass aber auch diese mit den Worten

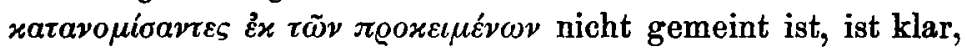
zumal das Vorstehende nicht einmal eine amtliche Ausfertigung eines älteren Erkenntnisses, sondern eine blosse, möglicherweise unrichtige Privatabschrift der vor Pechytes geptlogenen Verhandlung war.

Naber nimmt an, dass diese Schlusspartie einen von Pechytes herrührenden Anhang zu seiner Relation II 6-22 bildet;

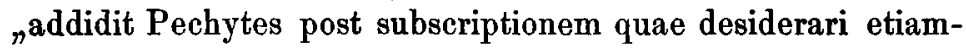
tunc intelligebat". Was Pechytes mit diesem Anhang bezweckt haben soll, ist mir nicht klar; auch ist es ganz unwahrscheinlich, dass eine Erklärung, die offenbar juristisch bedeutend ist - 'erkennend aus dem Vorstehenden, dass ...... schien es (uns), dass Panas rechtmälsiger Besitzer ist' die also eigentlich erst den Schlussstein seiner Amtshandlung bilden würde, von ihm als Postscriptum angehängt worden sei.

Mich dünkt am wahrscheinlichsten, dass die Schlussworte vom delegirenden Richter herrühren und als Enderledigung der vor Pechytes gepflogenen Verhandlung mitzitirt sind. Diese hatte, wie später zu erörtern, einen von den Parteien vereinbarten Conventionaleid des Panas und eine darauf folgende Anerkennung seines Rechts durch Thotortaios zum Erfolg; nun ist es in vielen Prozessordnungen vorgeschrieben, dass auf Grund solcher Parteianerkennung noch durch richterliches Urtheil (Agnitoria) dem Kläger sein Recht formal zuzuerkennen ist, und das mag auch hier vorliegen. Auch kommt in Betracht, dass das Anerkenntniss auf Grund des Eides erfolgte und durch diesen offenbar bedingt war; daher hatte es guten Sinn, dessen formellcorrecte Ablegung noch durch Urtheil zu constatiren. Wenn Naber bemerkt, dass ein Urtheil nicht mit żpaívezo (Col. 2 lin. 28), sondern mit paiveral im Präsens eingeleitet wird, so ist diese Bemerkung gegenüber einer blossen Agnitoria, welche sachlich keine neue Entscheidung bringt, sondern nur das bereits durch die Parteien Festgestellte wiederholt, 
noch weniger zwingend, als sie es schon an sich ist. Aber sie wiegt auch an sich nicht schwer; Urtheilsausfertigungen werden noch heute häufig auf das Präteritum 'das Gericht hat erkannt' gestellt.

Schliesslich ist noch zu bemerken, dass in Col. II lin. 23-24 noch auf eine andere Verhandlung in derselben Sache hingewiesen wird, welche vor dem gewesenen Oekonomen Dionysios stattgefunden hat. Man wird vielleicht die Vermuthung Nabers annehmen, wonach Panas, noch ehe er sich an Daimachos wandte, vorher schon bei Dionysios einen Prozess anhängig gemacht hatte, der übrigens in petitorio noch nicht zu Ende war, sondern erst zu einem Vorbescheid ( $\left.\delta \iota \xi \alpha \gamma \omega \gamma \eta^{\prime}\right)$, vielleicht auf Eidesleistung, geführt hatte und durch das zweite vor Daimachos eingeleitete Verfahren - die Einrede der Rechtshängigkeit mag dabei unberücksichtigt geblieben sein - sowie die in diesem letzteren Verfahren erfolgte Anerkennung sein Ende fand.

Wie endlich unser Papyrus zeigt, kam die Sache aber auch durch den zweiten Prozess noch nicht zur Rube, da Thotortaios trotz seiner Submission später neuerliche Ansprüche auf den streitigen Fleck erhebt. Dies bildet den Anlass zu der vorliegenden Eingabe, worin Panas unter Beifügung des von Pechytes und Daimachos Erhobenen um weiteren Rechtsschutz bittet.

Zum Schluss bemerke ich, dass die im Nachstehenden zu entwickelnden juristischen Folgerungen aus unserm Papyrus von der Auffassung dieses Punktes nicht unbedingt abhängig sind, sondern, wenngleich mit einer Einschränkung, auch von demjenigen gezogen werden müssten, der das Verhältniss der einzelnen Stücke sich in anderem Sinne zurechtlegt, als ich es gethan habe.

\section{II.}

Sein eigentliches Stigma erhält unser Papyrus durch

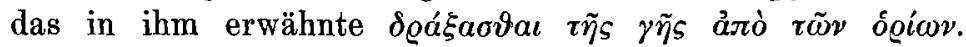
Man könnte, da, wie wir bald sehen werden, das $\delta \varrho a ́ \xi \alpha \sigma \vartheta a \iota$ $\tau \tilde{\eta} \varsigma \gamma \tilde{\eta} \varsigma$ mit der in den Quellen des classischen Griechenlands sich wiederholt findenden $\vec{\xi} \xi \alpha \gamma \omega \gamma \eta$ identisch ist, versucht sein, mit demselben noch jenen Passus in Col. II 24 zusammen- 


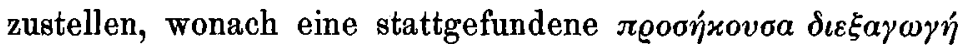
constatirt wird. Da jedoch diese letztere vor einem andern Beamten als Pechytes, nämlich dem gewesenen Oekonomen Dionysios stattgehabt hatte, und ausserdem $\delta \iota \varepsilon \xi \alpha \gamma \omega \gamma \eta \dot{\eta}$ sonst immer die Entscheidung schlechthin heisst, muss man es auf einen ältern Bescheid in dieser Sache beziehn, wie oben p. 282 geschehen ist, und das $\delta \varrho \alpha ́ \xi \alpha \sigma \vartheta a \iota$ allein in Betracht nehmen.

Worin aber bestand es? Wir müssen uns hier den Bericht über die Thätigkeit des Pechytes genauer ansehn.

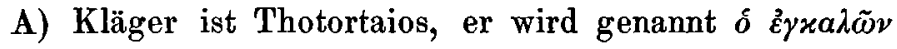
$\tau \tilde{\omega}$ Mavã $\iota$, und das gegen Panas eingereichte Libell, dessen in Col. 2 lin. 7 Erwähnung geschieht, geht offenbar von ihm aus. Er hat nun bei der Tagfahrt vor Pechytes den Vorschlag gemacht ( $\pi \varrho o \varepsilon \beta a ́ \lambda \varepsilon \tau o$ ), dass Panas (denn nur er kann in dem nachfolgenden Acc. c. Inf. das Subject sein) $\delta \varrho a \xi \alpha$ -

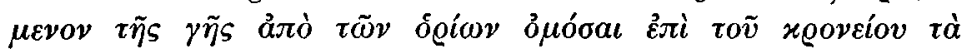

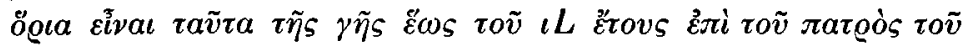

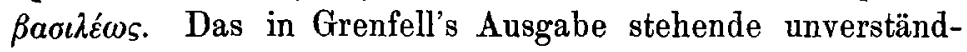
liche $\delta \varrho v \xi \alpha \mu^{\prime} \varepsilon v o v$ ist natürlich entweder verschrieben oder

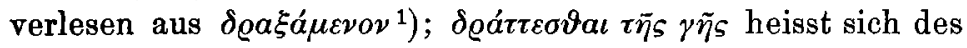
Besitzes am Boden bemächtigen und ist offenbar synonym mit demjenigen, was sonst $\dot{\varepsilon} \xi \alpha \dot{\alpha} \varepsilon \varepsilon v$ und auch in unserm $\mathrm{Pa}$ -

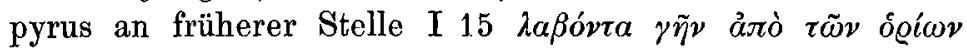
genannt wird. Der Sinn ist: Panas soll sich als Besitzer des Grundstücks geriren, indem er sich desselben - natürlich symbolisch - bemächtigt. Und zwar soll letzteres ge-

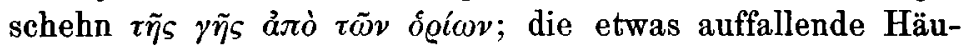
fung dieser Separativformen bezieht sich wohl darauf, dass es sich nur um einen Grenzstreifen handelt und die Bemächtigung eigentlich nicht an dem ganzen Feld, sondern nur an dem streitigen Streifen stattfindet. - Der Kläger provozirt ferner auf den Eid des Beklagten und dieser wird,

1) Diese einleuchtende Aufklärung gibt v. Wilamowitz in den Add. et Corr. des zweiten Bandes der Pap. Grenf.; ich darf hinzufügen, dass auch ein hier verweilender griechischer Gelehrter, Herr Dr. Pappulias aus Athen, dem ich die Stelle vorlegte, sofort und ohne diese Conjectur zu kennen, die gleiche Richtigstellung vollzogen und damit eine deutliche Bestätigung für die Natürlichkeit dieser Lösung gegeben hat. 
nach geschehener Bemächtigung (letzteres wird nicht besonders erwähnt, aber offenbar subintelligirt) auch von einem dazu eigens mitgegebenen Mann nach schriftlicher Eidesformel abgenommen. Dies Alles hatte natürlich eine besondere auf der streitigen Parzelle abgehaltene Lokalkommission zur Voraussetzung; doch scheint Pechytes der Unterrichter dabei nicht persönlich anwesend gewesen zu sein, da er sagt, er habe den Eidesempfänger "mitgeschickt". In der That war auch seine Anwesenheit nicht nothwendig; die Thätigkeit der Parteien lag ja offenbar gerade darin, dass sie die streitige Grenze genau bezeichneten, und er begnügte sich damit, dieselbe später, nach ihrer Rückkunft und ihren einverständlichen Angaben, d. h. jedenfalls nach den von ihnen ihm mitgetheilten Grenzmalen in abstracto abzumar-

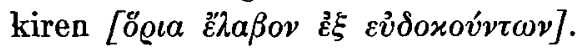

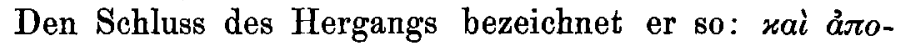

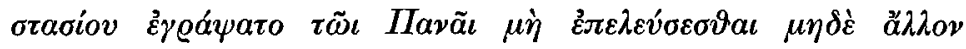

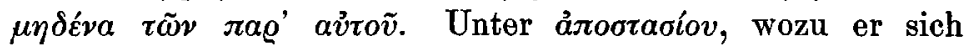

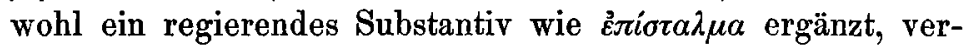
steht Grenfell ein Abstandsgebot („bill of ejectment") und meint, dass dieses sich gegen Panas gerichtet habe, indem

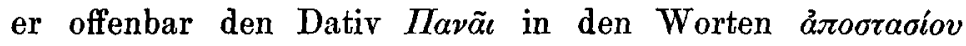

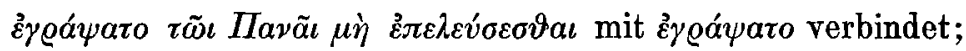
aber so sehr dies grammatisch naheliegt, ist es doch ganz unmöglich. Ich will davon absehen, dass Grenfell in Folge dessen den Panas als sachfällig ansieht, was nach dem von diesem erbrachten Eidesbeweis ausgeschlossen ist; man könnte ja immerhin noch daran denken, dass das Abstandsgebot nur ein prozessuales Interimisticum zu Gunsten des Thotortaios schaffen soll. Aber erstens wäre auch das kaum zu erklären, da Thotortaios, indem er das $\delta \varrho a ́ \xi \alpha \sigma \vartheta a \iota$ duldet, offenbar auch auf den Besitzanspruch verzichtet, und vor Allem: in Col. I lin. $20 \mathrm{f}$, wo nach Grenfell's eigener Angabe Panas es ist, der über jenen Termin berichtet ${ }^{1}$ ), ist deutlich gesagt, dass seinen Gegnern die Störung verboten war. Es bleibt daher nichts übrig, als $\Pi a \nu \tilde{a} \iota$ zu verbinden mit $\mu \grave{\eta} \dot{\varepsilon} \pi \varepsilon \dot{\lambda} \varepsilon \dot{\sigma} \sigma \sigma \vartheta a u$; das Gebot Ruhe zu halten richtet sich an

1) Nach meiner oben (S. 278 fg.) dargelegten Auffassung folgt dies schon daraus, dass der Gesuchsteller sich auf das Erkenntniss in Col. 2 1. $23 \mathrm{fg}$. beruft; dieses lautet aber zu Gunsten des Panas. 


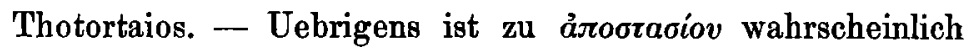

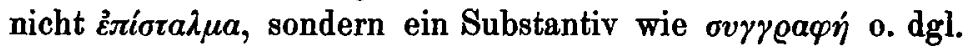
zu ergänzen und danach kein gerichtliches Decret, sondern ein Anerkenntniss des Thotortaios zu verstehn. Es findet sich nämlich, was freilich Grenfell seiner Zeit noch nicht

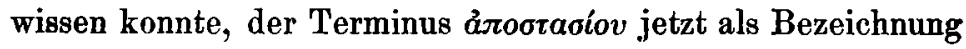
der Auflassungsurkunden über Grundstücke, welche zum Vollzug des Verkaufs ( $\pi \varrho \tilde{a} \sigma \iota \varsigma)$ bestimmt sind, vgl. Spiegelberg, Die demotischen Papyrus der Strassburger Bibliothek (1902) p. 10, Wilcken, Arch. f. Pap.-Forsch. S. 143. An unserer Stelle ist freilich, da Verkauf nicht vorliegt, an technische Auflassung nicht zu denken; wohl aber kann der Ausdruck den Verzicht auf weitere Besitzansprüche, also das Abstehn bedeuten, wie er ja auch in den eigentlichen ảro-

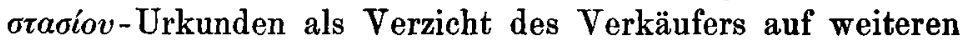
Besitz zu verstehn ist.

Mit dieser Anerkennung betrachtet Pechytes seine Aufgabe für erledigt und unterbreitet die Sache jetzt seinem

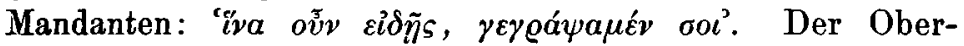
richter erklärt jetzt, wenn ich die Sache richtig deute (oben S. 280), dass Panas den Grenzstreifen zu Recht inne hat. Und zwar erklärt er dieses 'erkennend auf Grund des im Einvernehmen beider Theile von Panas abgelegten Eids', wobei offenbar das auch den Römern bekannte Institut des Conventionaleids (iusiurandum voluntuarium) zur Anwendung gebracht ist; die von Thotortaios noch dazu ausgesprochene

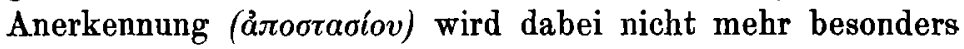
erwähnt.

B) Wenngleich aber nach diesem Entwicklungsgang des Prozesses für die Entscheidung der Wille der Parteien mafsgebend gewesen ist, so darf man deswegen doch nicht auch das $\delta \varrho a ́ \xi \alpha \sigma \vartheta a \iota$ als einen rein zufälligen und freiwilligen Act betrachten; vielmehr ist dieses ein nothwendiges Entwicklungselement des später freilich abgeschnittenen Prozesses. Hiefür spricht wie ich glaube die ganze Sachlage. Es hätte nämlich absolut keinen vernünftigen Sinn, dass Thotortaios vorschlägt, sein Gegner solle sich des Besitzes bemächtigen und dann schwören, wenn es sich bei Ersterem nicht um einen durch das Prozessritual gebotenen Formalact handelte. 
Denn das ganze Interesse des Thotortaios ist ja eben darauf gerichtet, dem Panas das Eigenthum streitig zu machen; wenn er nun, sei es im Bewusstsein der Schwäche dieser seiner Bestreitung, sei es aus anderweitigen Rücksichten, zum Beispiel aus ritterlichem Entgegenkommen, sich bereit finden lässt, die Entscheidung von einem Eid seines Gegners abhängig zu machen, so konnte doch der logisch und praktisch allein mögliche Inhalt seines Vorschlags immer nur der sein: Panas soll zuerst schwören und dann sich des Besitzes bemächtigen.

Der Vorgang des Thotortaios wäre unverständlich, wenn er so aufgefasst würde, dass er dem Panas zuerst schlechthin weichen und dann von ihm den Beweis seines Rechtes verlangen wollte. Demnach muss das, was er jenem einräumt, nur ein Provisorium oder Formale gewesen sein, und dieses Formale muss einen besondern Grund in der Anlage des Prozesses gehabt haben. Nur wenn wir diesen Grund als vorhanden annehmen, begreifen wir die Nachgiebigkeit des Klägers, der im Uebrigen ein sehr hartnäckiger Gegner war, wie seine wiederholten Angriffe auf die Stellung des Panas zeigen - denn er hatte schon einmal gegen ihn prozessirt - s. oben S. 282. Wir müssen darum annehmen, dass es sich um die Erfüllung eines für den Fortgang des Prozesses absolut erforderlichen Actes handelt und dass Thotortaios, indem er denselben in Anregung bringt, auf die Entwicklung des Prozesses hinarbeitet. Dass er dabei gleichzeitig seinen Gegner zum provisorischen Besitz zulässt, steht dem nicht entgegen; dazu wird er gewiss seine Gründe gehabt haben. Sehen wir doch in dem bekannten, unten noch zu erwähnenden Eigenthumsprozess zwischen Aebutius und Caecina über den fundus Caesennianus, dass Aebutius, dem es gleichfalls nicht minder ernst um die Sache zu thun ist, sich auf eine analoge Concession einlässt. Offenbar hatte eben Thotortaios in einem Streit über den factischen Besitz ebensowenig Aussichten auf den Sieg als Aebutius (unten p. 298 n. 1).

Seiner äusseren Form nach ist das $\delta \varrho \alpha ́ \xi \alpha \sigma \vartheta \alpha \iota$ ins $\gamma \tilde{\eta} s$ reiner Privatact; der Richter ist gar nicht dabei anwesend, sondern nur die Streittheile und die Zeugen. Pechytes er- 
wähnt ausdrücklich, er habe ihnen einen Commissar zur Eidesabnahme mitgegeben; also war er jedenfalls bei der ganzen Action nicht anwesend und der Commissar fungirt wieder nur bei der Eidesabnahme, nicht bei dem Vorhergehenden.

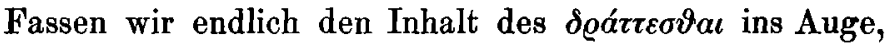
so ist klar, dass es eine Besitzergreifung des $\delta \varrho a \xi a ́ \mu \varepsilon v o \varsigma$ bedeutet. Dieses Besitzergreifen erfolgt aber höchst wahrscheinlich durch das Hinausführen des Prozessgegners; denn wie sollte sonst die Besitzergreifung in der für einen Formalact genügend plastischen Weise sich vollzogen haben? Und zwar erfolgt dieses Abführen hier im Einverständniss beider Theile. Sein Erfolg aber ist abgesehen davon, dass es als Erfüllung eines prozessualischen Formerfordernisses dem Prozess den weiteren Fortgang sichert - der Zweck hiervon wird alsbald zu erörtern sein - auch dieser, dass durch das $\delta \varrho a ́ \tau \tau \varepsilon \sigma \vartheta a \iota$ die streitige Parzelle identifizirt wird, und zwar allem Anschein nach unter genauer Feststellung des Grenz-

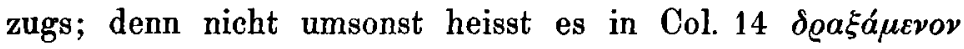
$\tau \tilde{\eta} \varsigma \gamma \tilde{\eta} \varsigma \dot{\alpha} \pi \dot{o} \tau \tilde{\omega} \nu \dot{o} \varrho i \omega \nu$. Vielmehr sind diese Worte sehr wesentlich; der Kläger bemächtigt sich des Streifens unter Markirung bestimmter Grenzen, auf denen er beim Wegstossen seines Gegners Posto fasst und darum wird denn

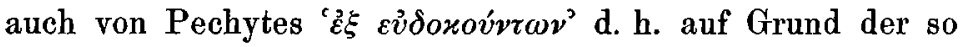
festgestellten und vom Gegner anerkannten Grenzansprüche des Panas die Abmarkung vollzogen.

Demgemäss können wir sagen:

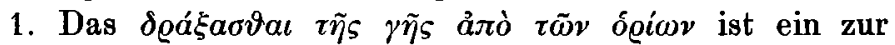
Durchführung des Prozesses über die streitige Parzelle erforderlicher Akt.

2. Es wird vorgenommen auf Vorschlag eines Streittheils, u. zw. in unserm Fall des Klägers, und im Einvernehmen beider Theile. Letzteres ergibt sich auch schon daraus, dass

3. es eine einverständliche Feststellung über die $\mathbf{A b}$ grenzung des controversen Feldstreifens bringt.

Diese Thatsachen sind nunmehr zu vergleichen mit den sonstigen über dieses Instjtut erhaltenen Nachrichten. Denn es wird wohl nicht als Petitio principii gelten, wenn wir die 
in den classisch-griechischen Quellen wiederholt erwähnte $\grave{\varepsilon} \xi \alpha \gamma \omega \gamma \dot{y}^{\prime}$ und ebenso die römische deductio quae moribus fit (= vis ex conventu) mit ihm zusammenstellen. Freilich sind die Nachrichten, die wir über beide besitzen, sehr dürftig; aber umsomehr muss jede Möglichkeit, das hier noch lagernde Dunkel aufzuhellen, begrüsst werden.

I. Die griechischen Quellen sind die (sogenannt) demosthenischen Reden gegen Zenothemis 17 sq. p. 887 und gegen Leochares 32 sq. p. 1090, dann Isaios de Pyrrhi her. 22 p. 28, 53, 62 und de Dicaeog. her. 22 p. 101, endlich Polybios 12 c. 16. Doch ist die Bedeutung dieser Stellen eine ungleiche, weil in einigen die Sache nur flüchtig und undeutlich berührt wird; wirkliche Belehrung ergeben nur die Rede gegen Zenothemis und die Stelle bei Polybios.

Das Fragment der Einrede gegen Zenothemis ist bislang wenig verstanden worden ${ }^{1}$ ); aber so schwierig es scheint, aus einer offenbar einseitigen Darstellung den wahren Thatbestand vollkommen zu ermitteln, lässt sich doch hier weiter kommen, als die philologischen Bearbeiter bisher gekommen sind. Es handelt sich um einen Getreideimport, den ein attischer Importeur (wer es ist, davon später) aus Syrakus bewerkstelligte. Zu diesem Zweck war ein eben im Peiraieus vor Anker liegendes Schiff nach Syrakus geschickt worden; dieses Schiff gehörte einem Massalioten, namens Hegestratos, in dessen Begleitung sich der jetzige Kläger Zenothemis befand, beides unverkennbar abgefeimte Halunken. Ausserdem wurde in Athen noch ein gewisser Protos eingeschifft, der den Einkauf in Syrakus und die Rückbegleitung der Fracht nach Athen besorgt hat, und von dem es zweifelhaft ist, ob er selbst der Importeur ist oder nur dessen Superkargo. ${ }^{2}$ ) - In Syrakus nun leitete der Schiffer Hegestratos in Verein mit Zenothemis eine ganz niederträchtige Specu-

1) Eine in Einzelheiten verständige Auseinandersetzung des schwierigen Rechtshandels gibt Philippi in den Neuen Jhb. für Philol. 95 p. $584 \mathrm{f}$; aber wegen mangelnder Schärfe in der juristischen Auffassung tritt die eigentliche Gestaltung des Rechtsfalls, welche eine vollkommen klare ist, nicht recht hervor. - ${ }^{2}$ ) Zweifelhaft ist und bleibt es aus dem einfachen Grunde, weil er selbst, wie sich später zeigen wird, über diesen Punkt zu verschiedenen Zeiten verschiedene Angaben macht. 
lation ein, indem sie dort bei einer massaliotischen Handelsfirma ein Seedarlehn auf das Schiff und die Ladung - die sie als ihr Eigenthum bezeichneten - aufnahmen, in der Absicht, das Schiff auf der Rückreise nach Athen in den Grund zu bohren; natürlich nahmen sie das geliehene Geld nicht mit, sondern schickten es gleich in ihre Heimath. Das Gaunerstück misslang, denn Hegestratos wurde, als er auf hoher See zur Nachtzeit in das Schiff ein Leck hauen wollte, noch rechtzeitig von der Bemannung abgefasst, stürzte sich, wie der Redner sagt, um der empörten Mannschaft zu entgehen, ins Meer - wahrscheinlich wurde er hineingeworfen - und ertrank. Die Besatzung suchte den nächsten Hafen - Kephallenia - auf. Dort erhob sich Streit über die Weiterfahrt. Denm jetzt suchte Zenothemis von dem gescheiterten Plan durchzuführen, was noch durchzuführen war; er wollte nämlich das Schiff nach Massilia führen, offenbar in der Erwägung, dass es dort wenigstens den Ansprüchen der attischen Befrachter leichter entgehen würde, und höchstens das Darlehn der syrakusanischen Firma, die ja in Massilia ihre Centrale haben musste, zurückzugeben sei. ${ }^{1}$ ) Indessen erhob der obengenannte Protos Widerspruch und, da er jedenfalls den Frachtvertrag Athen-Syrakus und zurück vorweisen konnte, wurde Zenothemis von der kephallenischen Seebehörde gezwungen, nach Athen zurückzufahren.

Und hier setzt nun der gegenwärtige Rechtshandel ein. Das Getreide wurde gelöscht und von Protos in Besitz genommen. ${ }^{2}$ ) Jetzt aber erhob Zenothemis Ansprüche darauf, mit der Behauptung, er habe dem Hegestratos auf dasselbe Geld geliehen; damit ist implicite die weitere Behanptung gegeben, dass dieser selbst das Getreide gekauft hatte. Der Anspruch des Zenothemis ist offenbar der hypo-

2) In dieser Action wurde er unterstuitzt, ja, wie der Redner merkwürdigerweise behauptet, sogar dazu angeregt, durch einen Agenten Namens Aristophon, welcher von den attischen Schiffsinteressenten, nachdem dieselben von dem Vorgefallenen gehört hatten - offenbar lag das Schiff längere Zeit im Hafen von Kephallenia - dorthin ge-

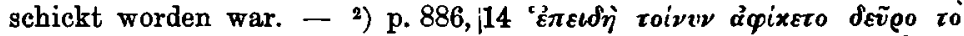

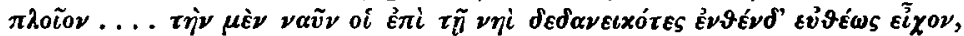

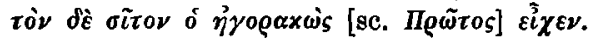

Zeitschrift für Rechtsgeschichte. XXIII. Rom. Abth. 
thekarische. Und nun tritt folgendes ein. Zenothemis erklärt, dass er sich von dem Pfand nicht durch Protos wegführen lassen wolle, sondern nur durch den Clienten des Redners, einen gewissen Demon. ') Was hat das zu bedeuten?

Der Sinn scheint mir klar zu sein. Zenothemis wollte seine Klage nicht gegen Protos richten, sondern gegen Demon - die Gründe kläre ich alsbald auf. Wie aber konnte er das? War nicht Protos im Besitz - der Redner sagt ja deutlich, dass er die Fracht in Empfang genommen hatte - und hat es einen Sinn, die Hypothekarklage gegen einen Nichtbesitzer zu richten? Nur weil die philologischen Bearbeiter wie begreiflich diesen Punkt nicht zu würdigen wissen, sind sie hier nicht weiter gekommen. Offenbar ist es eben die Meinung des Zenothemis, dem Protos den Eigenbesitz zu bestreiten; er behauptet, dass Protos nur Besitzvertreter des Demon und der juristische Besitzer der Fracht nur dieser letztere sei. Indem er dieses behauptet, stellt er sich natürlich auf den Standpunkt, dass Protos gar kein selbständiger Geschäftsmann, sondern blosser Agent des Demon ist.

Umgekehrt zeigt Demon das Bestreben, den Protos als Besitzer und selbständigen Unternehmer hinzustellen: er behauptet, dieser sei der wahre Importeur und habe sich von ihm, Demon, lediglich ein Darlehn zum Ankauf geben lassen. ${ }^{2}$ )

Protos selbst zeigt eine zweideutige Haltung; ursprünglich theilt er den Standpunct des Demon und will Besitzer des Getreides sein. ${ }^{3}$ ) Aber später, da die Getreidepreise plötzlich fallen, ändert sich seine Haltung und er tritt ganz auf die Seite des Zenothemis; wie der Redner behauptet,

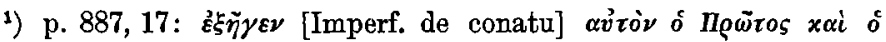

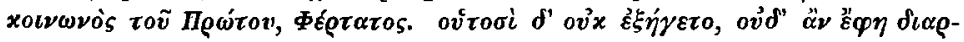

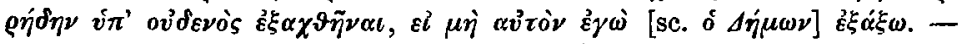

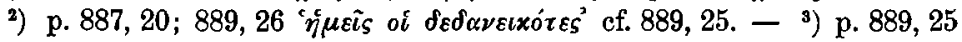

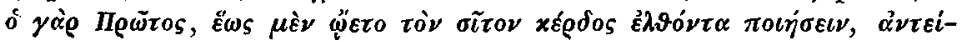

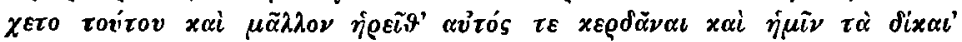

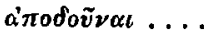


haben sie jetzt abgekartetes Spiel gemacht. ${ }^{1}$ ) Die praktische Rechnung aller drei Betheiligten ist unschwer zu durchschauen.

Protos calculirte so. Solange die Getreidepreise hoch standen, war es für ihn ganz günstig, selbst als Importeur des Getreides zu gelten. Er konnte dann gut verkaufen, konnte also dem Demon das geliehene Geld leicht zurückzahlen und noch einen hübschen Reingewinn einstreichen. Als aber die Preise fielen, sah er, dass er durch den Verkauf nicht einmal den Betrag des Darlehns herein bringen würde, sondern noch darauf zahlen müsste. Flugs erklärt er, das sei gar kein Darlehn gewesen, sondern - das ist offenbar die unausgesprochene Consequenz - bloss mitgegebener Betriebsfonds; er habe im Namen des Demon gekauft und brauche das Geld natürlich nicht zu zahlen, sondern nur zu verrechnen. Wird dann die gekaufte Fracht dem Demon von Zenothemis abgestritten, so geht ihn das folgerichtig nichts an. Nebstbei bekommt er dann von Zenothemis, dessen Betrug auf diese Weise ausführbar wird, eine Tantième.

Die Erwägung des Demon ist dadurch von selbst gegeben; er rechnet eben gerade umgekehrt und will dem Protos die Gefahr des Streits mit Zenothemis und später das Sinken der Curse aufbürden.

Endlich Zenothemis will sich einfach die Fracht aneignen; der Pfandcontract mit Hegestratos, auf den er sich beruft, ist offenbar erdichtet. Dabei erheben sich nun zwei Fragen.

a) Wie konnte Zenothemis auf Anerkennung seines Pfandrechts hoffen, nachdem doch das Getreide in Syrakus von Protos und nicht von Hegestratos gekauft war, worüber Protos die Quittungen hatte? Sehr einfach dadurch, dass er sich vornahm, diesen auf seine Seite zu ziehen und eine Production der Quittungen zu verhindern. Und so war es auch geschehen. Zenothemis war sogar noch weiter gegangen. Nachdem Protos die Farbe gewechselt hatte, hatte Zenothemis gegen ihn einen Scheinprozess auf Anerkennung seines

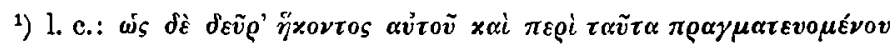

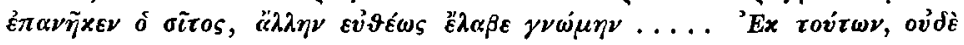

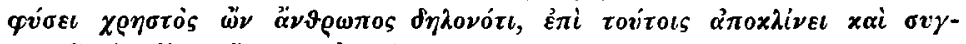

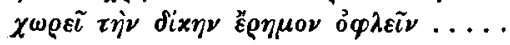


Pfandrechts angestrengt, Protos hatte verabredetermalsen das Land verlassen und war in contumaciam zur Anerkennung dieses Pfandrechts verurtheilt worden. ${ }^{1}$ ) Jetzt konnte Zenothemis allerdings hoffen, das Eigenthum des Hegestratos auch gegen Demon durchzusetzen, da ja dieser keinerlei Beweismittel über sein Eigenthum in Händen hatte.

b) Daraus ergiebt sich auch von selbst, warum Zenothemis früher, als Protos noch auf Seite des Demon stand, nicht gegen jenen, sondern gegen diesen klagen wollte. Eine Klage gegen Protos war eben ganz aussichtslos, weil dieser die Kaufsurkunden über das Getreide in Händen hatte; Demon war der Wehrlosere und ganz von der Unterstützung des Protos abhängig. Ausserdem war dieses Vorgehen ein eminent taktischer Zug insofern, als die Umgehung des Protos diesem von selbst zeigen musste, wo sein wahres Interesse lag: wurde Demon als der eigentliche Käufer hingestellt und er nur als der Agent, so brauchte er dem Demon das Darlehn nicht zurückzuzahlen und konnte dem ganzen weiteren Verlauf ruhig zusehen und das wird ihm beim ersten Zeichen eines Cursfalles sehr gut eingeleuchtet haben, worauf auch ein Passus in der Rede hindeutet. ${ }^{2}$ )

Nun ist der Rechtsfall soweit klargestellt, dass wir zur Betrachtung der $\dot{\varepsilon} \xi \alpha \gamma \omega \gamma \eta \dot{\eta}$ selbst übergehen können.

Thre Bedeutung ist jetzt von selbst klar: sie bestimmt die Passivlegitimation. Je nachdem Protos oder Demon sie vollzog, war die Hypothekarklage gegen den einen oder den andern zu richten.

Natürlich darf man sich aber dabei nicht vorstellen, dass jeder beliebige Nichtbesitzer die $\dot{\varepsilon} \xi \alpha \gamma \omega \gamma \eta \dot{~ v o l l z i e h e n ~}$ konnte; wenn Zenothemis sie von Demon verlangt, obwohl der körperliche Besitz des Getreides sicher bei Protos ist, geschieht das nicht in der Meinung, als ob man auch von einem Nichtbesitzer die $\vec{\varepsilon} \xi \alpha \gamma \omega \gamma \eta \eta^{\prime}$ erleiden könnte, sondern auf Grund der Behauptung, dass Protos nur Detentor und Demon der wahre Besitzer ist. Gerade weil er dies so lange als möglich bestreitet, weigert Demon die Vornahme der

1) S. die Stelle S. 291 N. 1. - ${ }^{2}$ ) Nämlich die in Anm. 1 S. 291

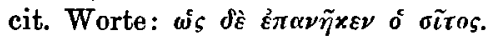


$\grave{\varepsilon} \xi \alpha \gamma \omega \gamma \dot{\eta}$, und als er sich schliesslich dazu bequemt, thut er es in der resignirten Erwägung, dass ihm rebus sic stantibus nichts übrig bleibt, als von Protos das Getreide zu „übernehmen ", also sich wirklich zum Besitzer zu machen. ${ }^{1)}$

Also: die $\dot{\varepsilon} \xi \alpha \gamma \omega \gamma \eta \dot{~ e n t h a ̈ l t ~ v o n ~ S e i t e ~ d e s s e n, ~ d e r ~ s i e ~ v o r-~}$ nimmt, das Bekenntniss des Besitzes und damit der Passivlegitimation.

Selbstverständlich muss es sein, dass der wahre Besitzer

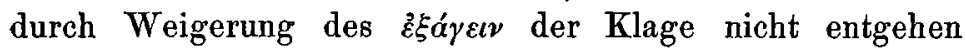
konnte; nur musste gegen ihn der Beweis des Besitzes er-

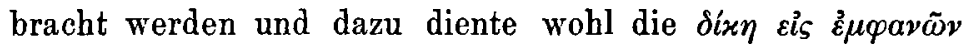

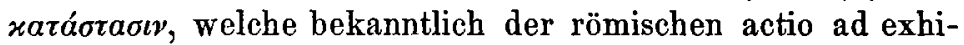
bendum entspricht. $\mathrm{Ob}$ er nach Erbringung dieses Beweises noch zu einem $\vec{\varepsilon} \xi \alpha \dot{\gamma} \gamma \varepsilon \nu$ gezwungen wurde, ist fraglich; bei Mobilien hätte dies jedenfalls keinen Sinn. Bei Grundstücken freilich blieb auch dann noch ein ganz verständiger Zweck über, wovon später zu sprechen ist, und in der Beschränkung auf Grundstücke bin ich geneigt, die $\stackrel{\varepsilon}{\xi} \xi \alpha \omega \omega \gamma \dot{\eta}$ für ein absolutes Formalerforderniss des dinglichen Prozesses anzusehen. -

Soviel ergiebt die Rede gegen Zenothemis. Ergänzend tritt hierzu der Bericht des Polybios 12, 16, über eine Begebenheit in Lokroi Epizephyrioi in Unteritalien.

Dort handelt es sich darum, welcher von zwei Männern, die um das Eigenthum eines Sklaven streiten, während des Prozesses den Besitz behalten und die Bürgen stellen soll. $A$ ist der ältere Besitzer, hat aber seinen Besitz gegenwärtig an $B$ eingebüsst, indem dieser den Sklaven gewaltthätig entführt hat. Dagegen hat $\mathbf{A}$ wieder den Sklaven aus dem Haus des B vor Gericht gebracht. Nun behauptet A, ihm gebühre der provisorische Besitz, denn

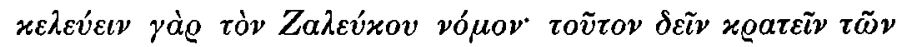

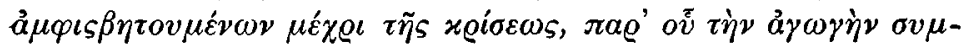

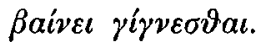

B dagegen beruft sich darauf, dass ja aus seinem·Haus die Wegführung vor das Gericht erfolgt ist. Der Kosmopolit,

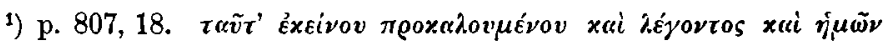

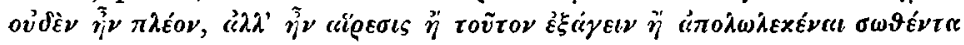

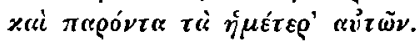


den man schliesslich befragt, erklärt, dass es nicht auf den absolut letzten, sondern auf den letzten rechtmälsigen Besitz ankommt

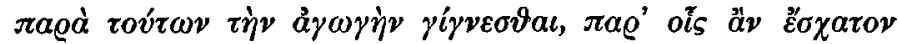

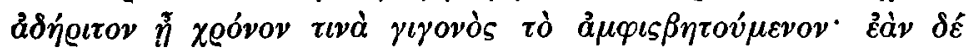

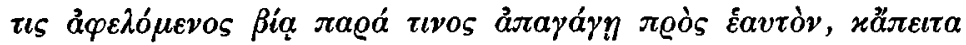

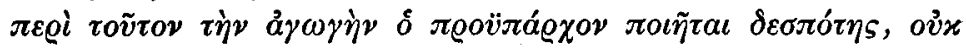

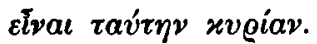

Es kann freilich auf den ersten Blick zweifelhaft sein, ob diese $\dot{a} \gamma \omega \gamma \eta^{\prime}$ mit der attischen sich deckt; es sieht fast so aus, als ob hier bloss auf die materielle Frage gesehen wäre, aus wessen Besitz die Sache vor Gericht gestellt worden sei (åy $\omega \gamma \dot{\eta}$ im Sinn der gerichtlichen Stellung) und F. Hofmann hat sich dadurch wirklich irre führen lassen. ${ }^{1}$ ) In Wahrheit ist aber auch hier ein Formalact gemeint, es

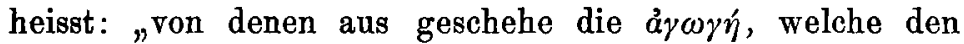
letzten unanfechtbaren Besitz gehabt haben." Schon das fällt offenbar mit der gerichtlichen Gestellung nicht mehr zusammen, weil diese auch von anfechtbaren Besitzern her erfolgen kann; und wenn man noch daran denken wollte, dass hier eine Fiction platzgreife und die vitiöse Besitzstörung selbst als $\dot{\alpha} \gamma \omega \gamma \dot{\eta}$ betrachtet werde, so entscheidet ganz

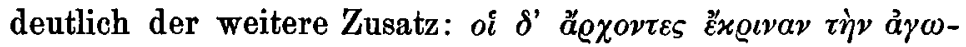

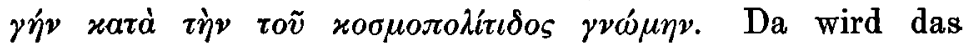
Recht der $\dot{\alpha} \gamma \omega \gamma \eta \dot{~ d e m ~ l e t z t e n ~ r u h i g e n ~ B e s i t z e r ~ z u e r k a n n t . ~}$ Möglich zwar, dass eine formelle Vornahme derselben, wenn die Sache schon vor Gericht war, unterblieb, obwohl ein Scheinact der Art auch jetzt noch ganz gut gesetzt werden konnte; aber jedenfalls konnte von einem solchen Zuspruch der $a \gamma \omega \gamma \eta \dot{n}$ nur die Rede sein, wenn sie auf einen formalen

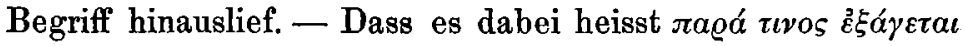
statt $\delta \iota a ́$ cıvos, begründet keinen Widerspruch zum attischen Recht; denn wenn in Attika sicher der Besitzer es ist, der

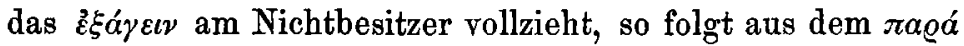
für Lokroi nicht, dass hier umgekehrt der Nichtbesitzer den Besitzer zu deduziren hatte. Denn $\pi \alpha \varrho \alpha ́$ bedeutet zunächst

1) Beiträge z. Gesch. d. griech. u. römischen Rechts 123, 126 ( so verschieden auch diese lokrische $\dot{\alpha} \gamma \omega \gamma \dot{\eta}$ von der attischen $\xi^{\xi} \xi \alpha \gamma \omega \gamma \dot{\eta}$ ist..."). 
nur räumlich den Ort, von wo aus die Deduction erfolgt, dieser ist beim Besitzer; dabei kann es sehr gut sein, dass auch er selbst der Deduzent ist. Ganz abgesehen davon, dass ein Historiker wie Polybios sich um solche Feinheiten nicht kümmert.

Das Positive, was wir aus Polybios erfahren, ist, dass die $\alpha \gamma \omega \gamma \eta \dot{y}$ ein Vorrecht des (letzten ruhigen) Besitzers bildet und vor Allem: dass sie nicht zum Besitz- sondern zum Eigenthumsstreit gehört. Ist der Besitz streitig, so ist es auch das Recht auf die $\dot{\alpha} \gamma \omega \gamma \eta^{\prime}$; sie ist in Bezug auf den Eigenthumsstreit der Ausdruck des Besitzes. Wird sie also im Einverständniss der Parteien vollzogen, so bedeutet sie, dass der Deduzirte damit den gegnerischen Besitz anerkennt und bereit ist, die Klägerrolle zu übernehmen.

Das sonstige in den Gerichtsreden noch vorfindliche Material ist durchaus minderwerthig, so dass daraus keinerlei Schlüsse gezogen werden können; es lässt sich sogar be-

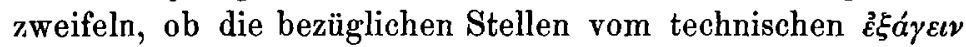
handeln. Beschränken wir uns demnach, die bei Polybios und im Zenothemisfragment gewonnenen Ergebnisse zusammenzustellen, so sind es diese.

1) Die $\vec{\xi} \xi \alpha \gamma \omega \gamma \eta$ ist ein Bestandtheil des Petitorium, nicht des Possessorium. Sie ist ein Recht des Besitzers, und solange die Besitzfrage nicht entschieden ist, ist auch nicht festgestellt, welcher Theil zu ihr befugt ist.

2) Wird die $\dot{\varepsilon} \xi \alpha \gamma \omega \gamma \eta \dot{\eta}$ im Einverständniss beider Theile vorgenommen, so enthält dies einerseits einen Verzicht des Weggeführten auf den Besitz und die Klägerrolle, andrerseits auf Seite des Wegführenden eine Anerkennung seiner Passivlegitimation.

3) Hieraus ergiebt sich gleichzeitig die praktische Bedeutung der $\bar{\varepsilon} \xi \alpha \gamma \omega \gamma \eta \dot{\eta}$; sie bewirkt eine Feststellung der Parteirollen. Dementsprechend scheint sie auch die regelmäfsige Einleitung des dinglichen Prozesses zu bilden. Zweifelhaft ist jedoch ihre Nothwendigkeit dann, wenn der Beklagte sie wegen mangelnden Besitzes verweigert hatte. $\mathrm{Da}$ in diesem Fall der Beweis seines Besitzes anderweitig erbracht worden sein muss, war hiemit wenigstens bei Mobilien alles geschehen, was sonst die $\vec{k} \xi \alpha \gamma \omega \gamma \eta \dot{~ z u}$ erbringen 
pflegte und es ist naheliegend, dass hiemit der Formalact, welcher nur noch eine leere Ceremonie hätte bilden können, entfallen sein wird. Nicht dasselbe ist aber für Immobilien anzunehmen, aus sofort zu erörternden Gründen.

Vergleichen wir damit die Ergebnisse des Papyrus, so stimmen sie damit aufs Beste überein. Auch hier findet sich eine Besitzergreifung am Streitobject - das $\delta \varrho a ́ \xi a \sigma \vartheta a \iota$

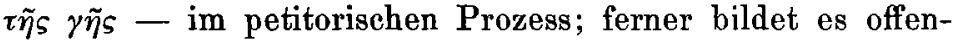
bar eine regelmälsige Voraussetzung desselben, da sonst der Prozessgegner sie nicht selbst vorschlagen würde. Dass sie im Einvernehmen beider Theile vorgenommen wird, zeigt dabei, dass der Kläger Thotortaios den Besitz des Beklagten nicht bestreiten konnte. Das Alles bietet dem classischen Stoff gegenüber nichts Neues. Aber einen Zuwachs erfährt das an diesem Gewonnene aus dem Papyrus durch die Beobachtung, dass die Streittheile bei dieser Gelegenheit auch, da es sich um ein Grundstück handelt, das streitige Stück Boden genau abmarkiren. Und hiemit gewinnen wir für den Immobiliarstreit eine neue hier höchst wichtige Function dieses Vorgangs; er dient dazu, bei Immobiliarprozessen den Streitgegenstand genau zu bezeichnen.

Es ist eine sehr richtige Bemerkung von A. Exner ${ }^{1}$ ), dass bei allen Grundstücksstreitigkeiten häufig die Parteien über die Grenze der beiderseitigen Rechtsbehauptung und damit über den eigentlichen Inhalt ihres Dissenses im Unklaren sind. Wenn zwei Bauern sich um ein Grenzstück streiten, weiss oft der Eine nur, wo er die Grenze haben will und dass der Gegner diese nicht annimmt; wo aber der Gegner selbst sie sucht, weiss er oft nicht. Ein solcher einseitiger Streit führt aber, wenn der Kläger mit seiner Behauptung nicht glatt durchdringt, zu keinem Ziel; gerade solche Streitigkeiten sind ihrer Natur nach zweiseitig und mit der Feststellung, dass der A zuviel verlangt, ist noch nicht gesagt, dass der $\mathrm{B}$ ihm genug zugesteht. Und das gilt nicht bloss beim reinen Grenzprozess zwischen Nachbarn; schliesslich ist auch beim Prozess über das ganze Grundstück es immer discutabel, wie weit dasselbe reicht,

1) In dieser Ztschr. 8 p. 179, $181 \mathrm{f}$. 
ja manchmal dissentiren die Parteien sogar über die Identität. Darum ist bei Grundstücks- und insbesondere bei Grenzprozessen ein localer Augenschein ein vorzügliches Mittel, um missverständlichem Prozessiren vorzubeugen und dem Prozess seinen Charakter als Definitivum zu sichern, und unsere Urkunde lehrt deutlich, dass die Griechen sich dessen klar waren. Und deswegen halte ich es auch für möglich, dass man diese zweite, bei Grundstücksprozessen neben der Feststellung der Parteirollen selbständig einhergehende Function der $\dot{\varepsilon} \xi \alpha \gamma \omega \gamma \dot{\eta}$ für unentbehrlich hielt und sie hier immer verlangte.

Ob dies Alles unser Urtheil über die römische deductio quae moribus fit zu beeinflussen vermag? Ich kann mir Manchen denken, der diese ganzen Ausführungen für lobenswerthe Excurse ins Griechische ansieht, im Uebrigen aber entschlossen ist, die bisher geübte Kunst der freien Composition auf dem Gebiet des älteren römischen Prozessrechts sich dadurch nicht verkümmern zu lassen. Prinzipiell anders steht die Sache für denjenigen, der an der ebenso biederen als bequemen Tradition von dem rein autochthonen Charakter der römischen Rechtsentwickelung irre geworden ist, wie es dem Verfasser dieser Zeilen in Folge vielfacher an anderem Ort in ihrer ganzen erdrückenden Menge zusammenzustellenden Beobachtungen ergangen ist; er wird sich gerade in Anwendung auf den dinglichen Prozess der Vermuthung nicht entschlagen können, dass die auffäligen Uebereinstimmungen, welche einerseits die römischen Besitzinterdicte mit dem in Gortyn und bei Polybios hervortretenden präparatorischen Besitzstreit, anderseits die vis ex conventu mit der $\bar{\varepsilon} \xi \alpha \gamma \omega \gamma \eta \dot{\eta}$ aufweisen, kein Zufall sind. Von dem Standpunkt ausgehend, dass in beiden Fällen jene Entlehnung aus der griechischen Gesetzgebung platzgegriffen hat, von der die Römer selbst eine so deutliche Erinnerung hatten, gelange ich zu der Ueberzeugung, dass der hier aufgedeckte Zweck der $\mathfrak{\varepsilon}^{\xi} \xi \alpha \gamma \omega \gamma \eta \dot{\eta}$ auch den Schlüssel zum Verständniss der deductio bildet. So wenig wir von dieser wissen, als feststehend ist zu betrachten, dass dieses Wenige 
dem oben gezeichneten Ductus des hellenischen Parallelinstituts in keiner Weise widerspricht. 1) Die deductio wird vollzogen nicht vom Kläger, sondern vom künftigen Beklagten - wobei freilich, wenn die Parteien über die Rollenvertheilung nicht einig waren - auch hier ein vorgängiger Besitzprozess entscheiden musste. ${ }^{1}$ ) 2) Die deductio ist Bestand theil des Eigenthums- (nicht des Besitz-) Prozesses; diese schon von Keller richtig erkannte und grundlos immer wieder bestrittene Wahrheit wird durch die Analogie des griechischen Rechtes, wonach das Recht auf die deductio erforderlichenfalls im Besitzstreit zu erkämpfen ist, diesen also schon voraussetzt, neuerdings bekräftigt. - Auch sonst lehren uns jetzt, was aus den ganz dürftigen und trümmerhaften Ueberresten des römischen Instituts nicht erkannt werden konnte, die griechischen Quellen kennen, nämlich den Zweck und Inhalt der im Einverständniss beider Theile vollzogenen deductio und dieser ist die formale Constatirung einerseits

a) dass derjenige, der sie an seinem Gegner vornimmt, die Passivlegitimation anerkennt, ferner

b) dass derjenige, der sich deduziren lässt, auf den Besitz verzichtet und den Besitz des Gegners anerkennt.

c) Bei Grundstücken kommt noch hinzu, dass mit der deductio eine Identifizirung der streitigen Fläche und die genaue Feststellung der beiderseitigen Besitzansprüche sich verbinden lässt.

Aus dem unter c) Gesagten geht gleichzeitig hervor, dass ich die vis ex conventu auch bei Mobilien für nicht a priori ausgeschlossen ansehe; erwägt man die Analogie des Zenothemisfragments und der Polybiosstelle und den Umstand, dass wir gar keinen Anhaltspunkt haben, ihre Be-

1) Darauf zielt der vielgedeutete Ausspruch Ciceros pro Caec. 1, 2: Nisi forte hoc rationis habuit (Aebutius) quoniam si vis facta esset moribus, superior in possessione retinenda non fuisset: quia contra ius moremque facta sit, A. Caecinam cum amicis metu perterritum profugisse sqq., das heisst frei übersetzt, wäre es zu einem Streit über das Vorrecht auf die deductio gekommen, so wäre Aebutius nicht in der Lage gewesen, sich im vorgängigen Besitzprozess die Rolle des deduzirenden Beklagten zu sichern. 
schränkung auf den Immobiliarprozess zu behaupten, so wird sich mindestens die Frage aufwerfen lassen, ob die deductio nicht ein viel weiteres als das gewöhnlich angenommene Anwendungsgebiet besass. Nur der Umstand, dass die Ciceronianischen Anwendungsfälle über diesen nicht hinausreichen, hat die heutigen Historiker dazu veranlasst, über dieses Gebiet nicht hinauszugehn. Allerdings aber bin ich weit entfernt, hier bestimmte Behauptungen aufstellen zu wollen; man muss sich durchaus gegenwärtig halten, dass man hier auf dem Boden der reinen Hypothesen stehen würde. Diese selbst aber, einmal ins Auge gefasst, führen von selbst zu weiteren Erwägungen. Wenn wirklich die Vindication im Sponsionsprozess einen an dem vindizirten Mobile vorzunehmenden Formalact erfordert (von dem wieder zu fragen wäre, ob er aussergerichtlich oder In Jure vorgenommen wurde), so wäre die nothwendige Consequenz nicht bloss, dass eine Mobiliarklage nur möglich war nach vorgängiger Feststellung des dem Beklagten gehörigen Besitzes, sondern auch, dass Fürsorge getroffen sein musste für den Fall, dass der Belklagte diesen ungehörig ableugnete, eine Schwierigkeit, die im Formularprozess der $\mathrm{f}^{\text {a }}$ petitoria nicht besteht, weil hier der Beweis des Besitzes erst im Prozess selbst zu erbringen ist. $\mathrm{Ob}$ sich von hier auf den ursprünglichen Zweck der doch auch heute noch räthselhaften actio ad exhibendum Schlüsse ziehen lassen, ist eine Frage, die ich hier allerdings nur aufwerfen kann; Stellungnahme zu den vielfachen und sehr schwierigen Problemen, die die römische Vindication heute noch bietet, wird nur auf Grund einer umfassenderen Darstellung alles Hierhergehörigen versucht werden können.

Dagegen bin ich geneigt, mich schon hier dafür auszusprechen, dass die Deductio dem Sponsionsverfahren ausschliesslich eigen war. Mit der legis actio sacramento in rem $^{1}$ ) scheint mir ein Vorgang, der die Besitzfeststellung

1) Für diesen Zusammenhang neuerlich Ubbelohde, Besitzinterdicte $641 \mathrm{fg}$., dessen Bemerkung 'eben weil es sich dabei (d. h. bei der deductio) nicht darum handelte, einen zweifellosen Besitzstand aufzugeben .... begreift es sich, dass jene Einigung ohne Schwierigkeit selbst unter den erbittertsten Gegnern vor sich ging' in ihrer offenbaren. 
zum eigentlichen Gegenstand hatte, kaum vereinbar, weil die dort stattfindende Zweiseitigkeit des Verfahrens und Vindizienverleihung zu der vorgängigen Regelung des Besitzes nicht passt. Dass die oft versuchte Verbindung der Deductio mit dem Besitzprozess') nach meiner Ansicht ausgeschlossen ist, braucht kaum bemerkt zu werden.

\section{P. Fir. 1.}

Unter den Papyrusurkunden, welche kürzlich für die Bibliothek des Istituto Superiore zu Florenz erworben worden sind, befindet sich ein Darlehns- und Hypothekarvertrag von ungewöhnlich guter Erhaltung, auf den die Leser dieser Zeitschrift hiermit hingewiesen werden sollen. Er wurde in trefflicher Lesung zuerst von $P$. Vitelli in der Zeitschrift Atene e Roma Jahrg. 1901 Nr. 27 herausgegeben; der Wiederabdruck der sehr umfangreichen Urkunde würde hier zu viel Raum beanspruchen, ich beschränke mich darauf, die auf die Hypothek bezüglichen Bestimmungen zu reproduziren. Nachdem die Bestimmungen des Darlehns - 200 Silberdrachmen zu $12 \%$ Zinsen auf 24 Monate - festgestellt sind, wird hinzugefügt, dass dieses Darlehn errichtet ist

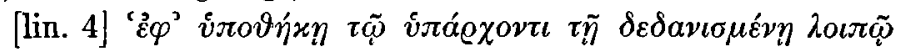

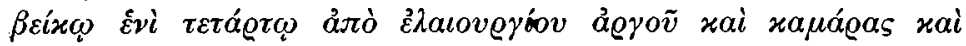

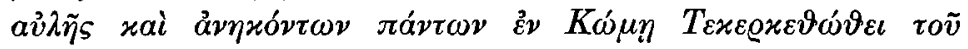

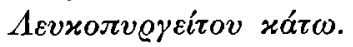

Ueber den Inhalt und die Geltendmachung dieser Immobi-. liarhypothek heisst es dann weiter lin. $6 \mathrm{fg}$.

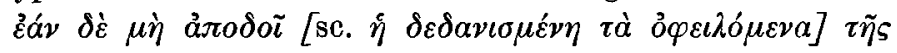

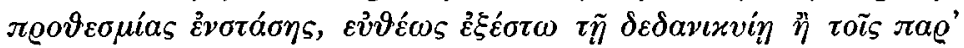

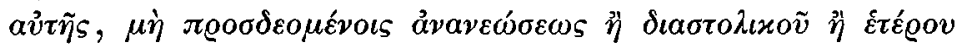

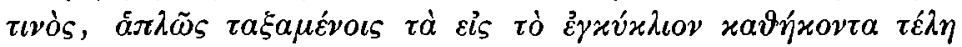

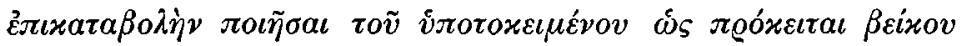

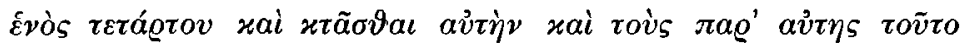

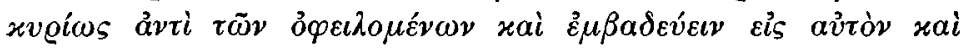

Unrichtigkeit ihn selbst am besten widerlegt. Ohne Besitzschutz weiss man nicht, wer das Recht zur Deductio hat; die Auffassung, dass, wenn der Besitzstand nicht zweifellos ist, die Parteien zu einer Einigung leicht geneigt sind, kann nur in der Gelehrtenstube aufkommen. Im praktischen Leben ist grade hier die Erbitterung am grössten.

1) So z. B. neuerdings wieder Pflüger, Besitzklagen 21-69 u. v. a. 


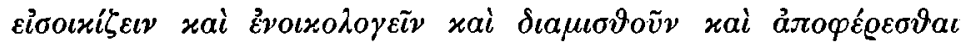

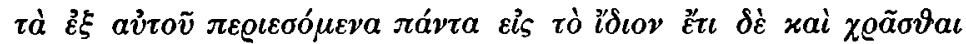

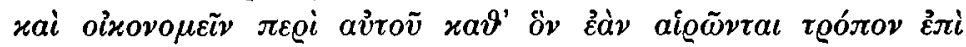

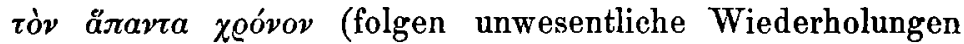
und die Bemerkung, dass die Verpfänderin gegen öffentliche und private Ansprüche garantiren muss).

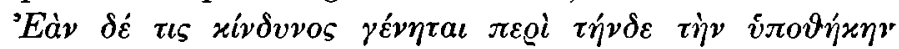

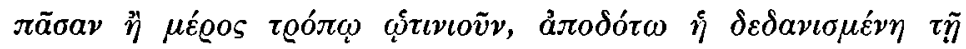

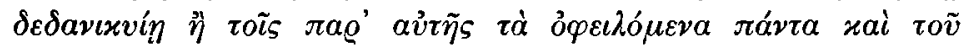

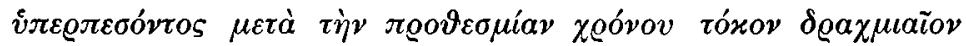

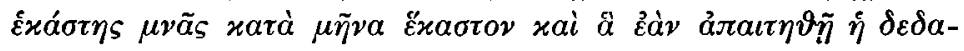

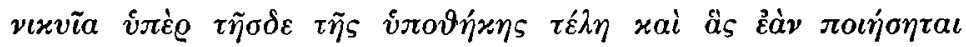

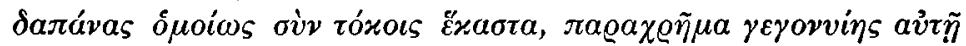

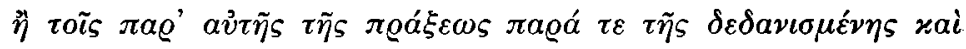

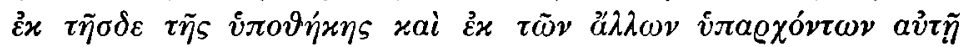

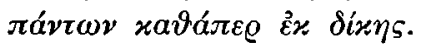

Die Hypothek ist ihrem Gehalt nach Verfallspfand, wie die griechische Hypothek überhaupt; wir kennen insbesondere in den Papyrusurkunden keinen Fall, wo sie in den Verkauf ausmünden würde. Die Folgen des Verfalles werden sehr ausführlich beschrieben; es tritt ein 1. $\dot{\varepsilon} \pi \iota \alpha \tau \alpha \beta \rho \lambda \dot{\eta}$ und $\dot{\varepsilon} \mu$ -

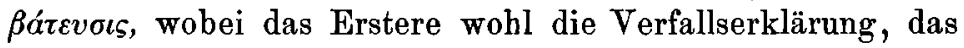
"Legen" des Pfands, das Letztere nach bekanntem Gebrauch die Besitzergreifung bedeutet. Damit verbunden ist 2. Uebergang des Eigenthums auf den Gläubiger; die unter den Bearbeitern des griechischen Rechts noch zweifelhafte Frage,

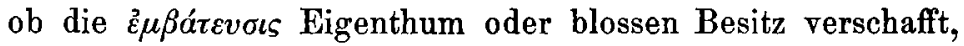
kann als durch diese Urkunde, sowie durch P. Oxy. 2, 270 entschieden angesehen werden; denn beide Mal wird dem Gläubiger Gebrauch und Verfügung über das ergriffene

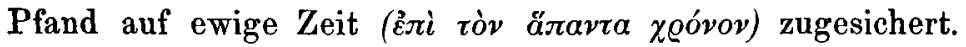

Dies ist bereits von anderer Seite bemerkt und ebenso ist betont worden, dass in den Papyri der Gedanke anklingt, dass mit der Pfandsetzung die persönliche Schuld getilgt sei. ${ }^{1}$ ) So wird in BGU 741 den Gläubiger zugesichert, dass er im Fall der Nichtzahlung sich an die Hypothek halten und ausserdem noch den nicht gedeckten Ueberschuss der Schuld mit

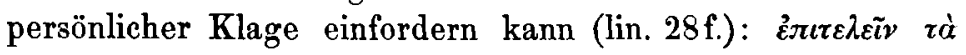

1) E. Costa, bull. dell' Istit. 1902. Vgl. Gradenwitz, Einführung 93. 


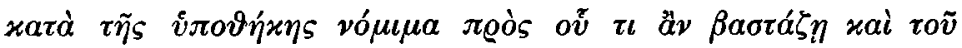
$\dot{\varepsilon} \nu \lambda \varepsilon i \psi$ iv wird, deutet zurück auf einem Rechtszustand, wo das Pfand gewissermalsen um die Forderung bedingt gekauft war, sein Minderwerth also auf Risico des Gläubigers ging. Mehr noch: in beiden Urkunden, BGU 741 und unserm Papyrus behält der Gläubiger sich vor, bei zufälligem Untergang der Hypothek auf seine Forderung zurückgreifen zu können, eine Erscheinung, die auch in einem sehr bekannten Rescript des justinianischen Codex anklingt.

Bis hieher erfahren wir nichts Neues. Ein Novum aber ist die Bestimmung in lin. $6 \mathrm{f}$, die Gläubigerin soll ihr

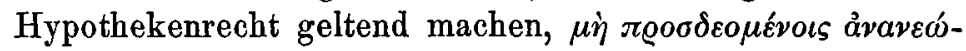

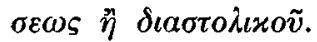

Unter den beiden Erfordernissen, welche hier der

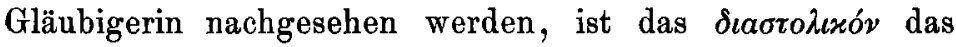
leichter zu verstehende; es bedeutet wahrscheinlich ein Einweisungsdecret. Der Ausdruck begegnet auch in BGU 832 lin. 25; in diesem leider wegen linksseitiger Zerstörung un-

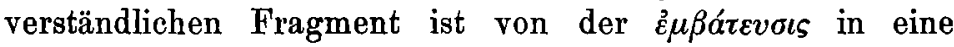
Hypothek die Rede $u$. z. in einer gerichtlichen Verhand-

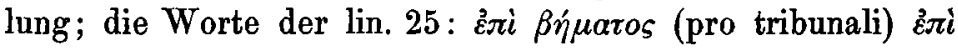

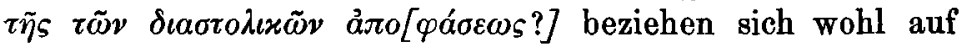
ein Begehren um die Einweisung in die Hypothek. Die Pfandrechtsklage ist in den Papyri auch noch an einer andern Stelle gegeben, nämlich in BGU 888, wo der Gläubiger vor Gericht verlangt, dass der Schuldner entweder zahlen oder ihm die Execution in seine Pfänder gestattet werden soll; freilich handelt es sich dabei vielleicht nicht um eine Hypothek im eigentlichen Sinn. - In unserm Fall ist die Frage nur die, ob der Wegfall des Einweisungsdecrets ausdrücklich ausbedungen werden musste, oder ob nur das Selbstverständliche betont wird; m. a. W. ob die Erhebung der Hypothekenklage an sich vorgeschrieben war und nur bei besonderer lex conventionis entfiel, oder ob sie schon nach dem Wesen der Hypothek entbehrlich war. Gegen letzteres könnte man sich etwa

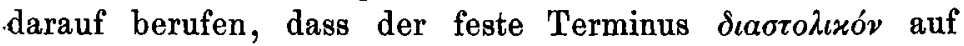
regelmäfsigen Gebrauch solcher Decrete hindeutet; doch 
ist dies nicht entscheidend, weil der Gläubiger oft freiwillig auf Eigenmacht verzichten mochte und auch durch Widerstand des Schuldners gegen die $\vec{\varepsilon} \mu \beta a ́ t \varepsilon v \sigma \iota s$ zur Erbittung gerichtlicher Einweisung gezwungen werden konnte. Immerhin ist zu bemerken, dass unsere Urkunde die Executivclausel

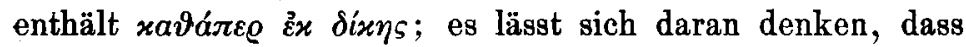

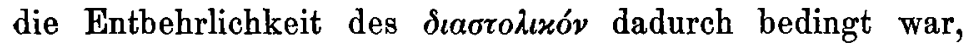
dass dem Gläubiger Executirrechte eingeräumt wurden. Aber eine sichere Entscheidung ist nicht zu gewinnen.

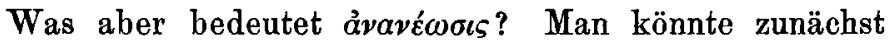
an eine Prolongationsfrist oder daran denken, dass dem Schuldner ex lege binnen einer bestimmten Zeit nach der Embateusis auch ein Wiedereinlösungsrecht zustand, etwa wie bei der Impetratio dominis. Aber beides ist dadurch

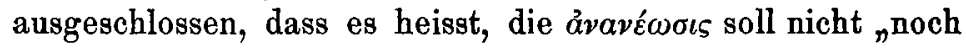
dazu erforderlich" ( beziehen auf eine vom Gläubiger vorzunehmende Handlung zur Ausübung oder Erhaltung seines Hypothekenrechtes.

Nun kommt aber der gleiche Ausdruck auch vor in dem Katasterfragment P. Oxy. R. 274, auf welches ich an anderm Ort schon vorübergehend hingewiesen habe. Dort ist für einen gewissen Sarapion eingetragen ein Hypothekenrecht an einem Haus und Hof und an diese Eintragung schliesst sich von zweiter Hand offenbar später geschrieben der Vermerk: Im $12 \mathrm{Jahr}$ am $5^{\text {ten }}$ Schalttag (28. August) hat Sarapion

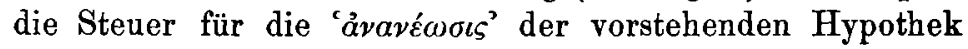
bezahlt. Und darauf von dritter Hand: 'Im $13 \mathrm{Jahr}$, am $5^{\text {ton }}$ Schalttag (28. August) hat Sarapion die gebührende Steuer der Hypothek bezahlt.' Beide Steuern sind nicht identisch; die erstere wird für den besondern Act der ảva-

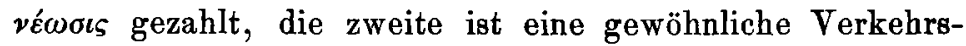
steuer, welche, wie wir aus dieser Stelle sehen, für die Hypothek nicht bloss bei ihrer Begründung, sondern auch während ihres Bestandes in gewissen uns nicht bekannten Zwischenräumen zu zahlen war. - Der Begriff der ảvavéwoıs selbst aber ist gerade an dieser Stelle ganz sicher zu bestimmen aus P. Oxy. 237 al. VIII lin. 40-43, wo der Statthalter Mettius Rufus behufs Evidenthaltung der Immobiliarrechtsverhältnisse vorschreibt, dass die Kataster, welche 
Eigenthum und Hypotheken ausweisen, alle fünf Jahre er-

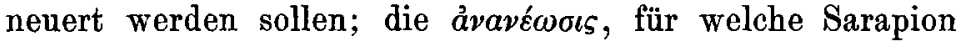
die Steuer bezahlt, ist eben eine solche Erneuerung des Katasters.

Und jedenfalls das Gleiche ist in unserm Hypothekarinstrument gemeint. Der Gedanke ist der, die Gläubigerin soll ihre Hypothek behalten, auch wenn sie für die Erneuerung im Kataster zu sorgen unterlässt. Dass das gesagt werden kann, ist freilich auffallend, weil die Fassion zum Kataster publizistische Pflicht ist; aber es ist begreiflich, dass die etwaige Versäumung derselben der privaten Berechtigung keinen Eintrag thun kann, sondern nur eine Verantwortung gegenüber der Behörde hervorruft und vor Allem, es ist zu beachten, dass es sich hier nicht um ein auf lange Dauer berechnetes dingliches Recht handelt - in diesem Fall hätte die Gläubigerin schon im eigenen Interesse die erneute Eintragung angestrebt - sondern um ein kurzfristiges. Da nämlich das Darlehn auf nur zwei Jahre läuft, so konnte die Darleiherin schon nach zwei Jahren ihre Hypothek realisiren; da lag es wirklich sehr nahe, wenn etwa die Neuanlegung wenige Monate vor dem Verfall des Pfands eintrat, sie von einer erneuten Fassion und der damit verbundenen Steuerzahlung zu dispensiren und zu bestimmen, dass sie ihre Hypothek solle auf Grund des alten Katasters fernerhin geltend machen können.

\section{D. 34, 9, 16 pr.}

Es sei mir gestattet, in diesem Zusammenhang die Erläuterung einer Digestenstelle vorzulegen, über welche schon seit Jahrhunderten die Ansichten stark auseinander gehen. Allerdings hängt die Erklärung derselben mit den Papyri nur lose zusammen, da für ihren wesentlichen Inhalt nach wie vor aus den römischen Quellen allein die Erklärung gesucht werden muss. Aber an einem Punkt lässt sich doch die Verbindung herstellen und das mag es rechtfertigen, dass ich sie bei dieser Gelegenheit erörtere.

D. 34, 9, 16, 1, Papinianus libro 8 respons.

Quoniam stuprum in ea contrahi non placuit, quae se non patroni concubinam esse patitur, eius qui concubinam 
habuit quod testamento relictum est, actio non denegabitur. Idque in testamento Cocceji Cassiani clarissimi viri, qui Rufinam ingenuam honore pleno dilexerat, optimi maximique principes nostri iudicaverunt; cuius familiam, quam alumnam testamento Cassianus nepti coheredem datam appellaverat, vulgo quaesitam apparuit.

Diese Ausführung Papinians hat viele Interpreten gefunden; eine Zusammenfassung verschiedener Auslegungsversuche findet sich bei Glück 28, $382 \mathrm{f.}^{1}$ ) Glücklich kann man diese Interpreten allerdings zum grössten Theil nicht nennen; selbst bei den bessern finden sich schwere Missverständnisse, manche werden geradezu willkürlich und gelegentlich noch abgeschmackt. Letzteres ist z. B. der Fall bei der von Accursius angenommenen Glosse des Vivianus, wonach als Processpartei nicht etwa die Vermächtnissnehmerin Rufina, sondern ihre allerdings zur Theilerbin eingesetzte unbenannte Tochter zu denken ist; diese Tochter soll ursprünglich Sklavin des Cassianus gewesen sein - wie das zugeht, nachdem doch die Mutter als ingenua bezeichnet wird, weiss Vivianus selbst nicht - ; später, da sie heranwächst und 'multum pulchra' wird, begehrt Cassianus auch sie zur Concubine, obwohl er schon mit ihrer Mutter im Concubinat lebt; das brave Mädchen aber, feinfühliger wie Cassianus, lehnt dies $a b$ und Cassianus begnügt sich sie zu seiner Erbin einzusetzen, eine Erfindung, die man zwar bei einem schlechten Romanschriftsteller, nicht aber bei einem Juristen zu begegnen gefasst ist. Weniger tugendhaft, aber im Uebrigen wo möglich noch schlechter ist die Interpretation des Cuiacius obs. 5, 6; nach ihm lässt sich die Tochter nach dem Tod ihrer Mutter (die als Ehefrau des Cassianus betrachtet wird) thatsächlich auf den Concubinat ein, was freilich nicht Wunder nehmen darf, da sie schon früher 'corpore quaestum fecit', eine 'Thatsache', die Cujaz dadurch herstellt, dass er die Worte 'vulgo quaesitam apparuit' ohne jeden Anhalt in den Handschriften in 'vulgo quaestum fecisse

1) In neuerer Zeit hat Leist (bei Glück, Serie der Bücher 37-38 $\checkmark$ p. 165) die Stelle, aber leider nur im Vortibergehen, berührt. In Meyers Schrift über den römischen Concubinat wird, wie es scheint, die Glück'sche Exegese angenommen (p. 162 n. 115). 
apparuit' verdreht. Diese Textcorruptel haben nun die späteren Ausleger abgelehnt und erkennen auch richtig, dass Rufina noch gar nicht gestorben ist und dass es sich um ein zu ihren Gunsten errichtetes Vermächtniss handelt; aber auch bei den besten ron ihnen, wie Pancirolus, de Retes, Merillius und Heineccius, bleibt der Gedanke bestehn, die Rufina gelte doch als eine mulier vulgaris, weil ihre Tochter vulgo quaesita war; sie habe also den Cassianus gar nicht heiraten können, und eben darum sei sie, der Praxis des Julisch-Papischen Gesetzes entsprechend, mit Recht und ohne stuprum seine Concubine. Aber auch diese noch von Glück acceptirte Auslegung ist ebenso willkürlich wie widerspruchsvoll.

Denn erstens ist nicht mit einem Wort darauf hingedeutet, Rufina sei wirklich eine mulier famosa im technischen Sinne des Worts und im Sinn insbesondere der lex Iulia et Papia gewesen. Dass dies der Fall sei, erschliesst man nur durch die sonderbare Verwechslung der unehelichen Conception mit Prostitution; in der That findet sich die Rufina bei jenen Gelehrten ohne weiteres bezeichnet als eine 'quae corpore quaestum fecerat' und Glück fügt erklärend hinzu: 'denn ihre Tochter wird rulgo quaesita genannt'. Dass das eine ganz plumpe Gleichstellung sehr verschiedener Dinge bedeutet, hätten aber ausser dem einfachen Menschenverstand auch die Quellen sagen können; in D. 23, 2, 43, 1-3 ist ganz ausdrücklich gesagt, dass noch nicht jedes aussereheliche Verhältniss ein pa la m corpore quaestum facere bedeutet, wenn auch solche Frauen im weiteren Sinne 'probrosae' sind, D. 23, 2, 41.' ${ }^{1}$ Damit stimmt überein, dass eine Frau, die bereits uneheliche Kinder hat, ganz wohl verheiratet sein kann, wie das Papinian in D. 31, 77, 2 voraussetzt; es ist in keiner Weise nothwendig, hier an die Ehe mit einem an die bezügliche Bestimmung des Julischen Gesetzes nicht gebundenen Libertinus zu denken.

Zweitens aber kommt bei dieser Ansicht der Widerspruch heraus, dass Rufina, die wegen ihrer Infamie einen Ingenuus nicht heiraten kann, doch sein Vermächtniss be-

1) Darüber unten p. 307 n. 1. 
kommt, während bekanntlich Domitian alle mulieres probrosae als incapaces erklärt hatte. ${ }^{1}$ ) Was hat es denn dann für einen Sinn, dass Papinian sagt, in ihrem Concubinat liege kein Stuprum, wenn sie doch von früher her mulier famosa und also doch incapax war? Grade das was der Jurist auf der einen Seite eliminirt, tragen die Interpreten von der andern wieder hinein, und was das Auffallendste ist, ohne es auch nur zu merken. So findet sich bei Glück p. 384 die richtige Bemerkung, die Rufina hätte vom Cassianus keine letztwillige Zuwendung erhalten können, wenn das Concubinat stuprum war, denn dass eine stupro cognita .... auch nicht aus einem letztwilligen Soldatentestament erben konnte, hat keinen Zweifel'. Auf p. 385 heisst es aber 'Unsere Rufina war zwar eine ingenua, aber doch eine mulier vulgaris quae corpore quaestum fecerat'.

Da gegenüber solchen Auslegungen wenig Ehre zu holen ist, will ich mich begnügen, das ganz ungenügende Verständniss dieser Stelle hier constatirt zu haben und zu einer positiven Darstellung übergehn, wonach die Stelle gleichzeitig einen lehrreichen Einblick in die Praxis des römischen Eherechts verstattet. Der Senator (vir clarissimus) Coccejus Cassianus hat eine gewisse Rufina mit einem Vermächtniss bedacht; dies liegt offenbar in den mit echt papinianischer Prägnanz comprimirten Worten 'eius qui concubinam habuit quod testamento relictum est actio non denegabitur. Ein Anderer würde freilich 'ei non denegabitur' gesagt und damit angedeutet haben, dass die Concubine die Bedachte ist; aber Papinian verschmäht jeden überflüssigen Buchstaben und darf es hier thun, weil, solange Niemand anderer als der Testator und seine Concubine genannt sind; auch nur an diese letztere

1) Sueton. Domitianus c. 8 'probrosis feminis lecticae usum ademit iusque capiendi legata hereditatesque'. 'Capere' ist hier technisch gesagt; der Inhalt der Bestimmung ist, dass diese Frauen, obwohl von der Ehe (mit Freigebornen) ausgeschlossen und daher eigentlich von der Cölibatsstrafe frei, doch von nun an derselben, wie die in freiwilligem Cölibat lebenden, unterliegen sollen (Savigny, Syst. 2, 557). Die älteren Interpreten (und auch jetzt noch Voigt, RG. 2, 711) nehmen hier vielfach Erbunwürdigkeit an; aber schon die Analogie von D. 29, 1, 41, 1 zeigt, dass an wirkliche Incapacität zu denken ist. 
als Legatarin gedacht werden kann. Dass nicht von der später erwähnten Erbeinsetzung der Tochter der Rufina die Rede ist, ist ausserdem ganz selbstverständlich schon deswegen, weil der beigegebene Grund, dass Concubinat kein Stuprum ist, eben nur für die Person der Rufina selbst einen Sinn hat. -

Dieser Rufina wurde das Vermächtniss wegen der Incapacität der 'mulieres probrosae' bestritten 1), da sie mit Cassianus im blossen Concubinat gelebt hatte. Letzteres bedeuten die Worte 'qui Rufinam honore pleno dilexerat', bezüglich deren es sprachwidrig ist, sie mit Cuiaz auf die Ehe zu beziehen und sinnwidrig, sie mit Perennonius in eine 'non honore pleno' zu verschlimmbessern. Gerade der plenus honor war es, der hier den Concubinat von einem Stuprum unterschied. Wäre freilich Rufina eine Freigelassene des Cassianus gewesen, so bedurfte es des honor dilectus nicht; mit seiner eigenen Freigelassenen ist dem Patron nach anerkannter Praxis immer der Concubinat erlaubt, er mag als offenes und ehrbares Verhältniss oder als lichtscheues Einverständniss erscheinen. Bei der Ingenua ist es anders: hier ist theoretisch sogar jeder Umgang stuprum und wenn davon die Praxis nach dem Muster des Privatlebens einzelner Regenten ${ }^{2}$ ) allmälig Ausnahmen anerkannt hat, so konnte sich das immer nur auf solche Verhältnisse beziehn, wo nicht schon in der Verstohlenheit des Verkehrs die Anerkennung seiner Unsittlichkeit und die Herabwürdigung der Frau zur Dirne gelegen war.

Nebenbei bemerkt sind diese Ausnahmen nicht von allen Theoretikern anerkannt worden; wenn Ulpian in D. 25, 7, 1, 1 schreibt 'Cum Atilicino sentio et puto solas eas in concubinatu habere posse sine metu criminis in quas stuprum non committitur, so zeigt er, dass es noch zu seiner Zeit wenigstens einen Juristen gab, der an dem allgemeinen

1) Von wem, ist nicht gesagt; es kommt wohl zunächst die eingesetzte Enkelin des Testators in Betracht. Andere denken an den Fiscus; aber damit stimmt der Ausdruck 'actio non denegabitur' nicht. überein. - ${ }^{2}$ ) So hatte Vespasian (Suet. Vesp. 3) eine Freigelassene der Antonia Augusta, Mark Aurel (vita Marci 29), die Tochter des Procurators seiner Frau, vielleicht sogar eine Ingenua, zur Concubine. 
und ausnahmslosen Wortlaut des Julischen Keuschheitsgesetzes festhielt. Aber dass Ulpian sich hiefür auf Niemand anderen zu berufen weiss als auf den um fast anderthalb Jahrhunderte älteren Atilicinus, zeigt auch, dass es sich hiebei um einen reinen Doctrinarismus handelt, der auch in der vorsichtigen Form seiner Behauptung zu Tage tritt. Ganz anderer Anschauung ist denn auch Marcian, der D. 25, 7, 3 pr. mit den Worten in concubinatu potest esse et aliena liberta et ingenua et maxime ea quae obscuro loco nata est vel quaestum corpore fecit' sicher der laxeren Anschauung huldigt, wobei noch dazu der Schluss 'et maxime .... fecit' zweifellos interpolirt ist. ${ }^{1}$ ) Bedenkt man noch, dass nach Paulus D. 25, 7, 5 der Provinzialbeamte sich eine Concubine aus seiner Provinz nehmen darf, wo doch schwerlich bloss an Freigelassene, Prostituirte und Peregrine gedacht ist, dass ferner Marcellus D. 23, 2, 41, 1 zwar der Concubine (dafern es nicht die eigene Freigelassene ist) die 'matris familias honestas abspricht, aber doch kein Stuprum statuirt, und hält man endlich dazu die Lebensführung der im offenen Concubinat lebenden Kaiser und die Selbstverständlichkeit, womit das gleiche Verhältniss auf den Inschriften behandelt wird ${ }^{2}$ ), so kann kaum ein Zweifel sein, dass die Regel des Ulpian von Niemand ernst genommen wurde, und Papinian in unserm Fall spricht das als ein praktisches Axiom aus (placuit), womit auch seine alsbald zu besprechende Entscheidung in D. 39, 5, 31 pr. übereinstimmt. Für dieses Axiom kann er denn auch ein Decret yon Severus und Caracalla beibringen; dass Ulpian auf dieses keine Rücksicht

1) Dass mit den obseuro loco natae der Concubinat „besonders leicht" möglich ist, ist erstens juristischer Nonsens - wie denn das 'et maxime' ganz unclassisch lautet - und zweitens entspricht diese Bestimmung zu deutlich den Gesetzen Constantins und Valentinians (C. Th. 4, 6, 3; C. I. 5, 5, 7), um nicht mit Rücksicht hierauf für interpolirt zu gelten. Zwar gelten die Laden- und Schankmädchen schon in der classischen Zeit (Paul. sent. 2, 26, 11) als kein Object des Stuprum, aber dieser Satz zeigt auch, dass man die Regel auf diese beschränkte, und dass jedes Frauenzimmer der niedern Stände zum Concubinat prädestinirt sei, ist späte Anschauung. - 2) Das Material hat Meyer a. O. gesammelt. Ueber das Einzelne und insbesondere den Soldatenconcubinat, sowie sein Verbältniss zur Ehe ist hier nicht zu handeln. 
nimmt, ist auffallend, aber bei einem einzelnen Decret doch ein begreifliches Uebersehen ${ }^{1}$ ), zumal dasselbe über die Frage des Stuprum nicht ex professo, sondern nur incidenter anlässlich eines Erbstreits entscheidet.

Also der Concubinat ist an sich auch mit der Ingenua denkbar; aber nicht jeder Verkehr ist ein solcher und es bleibt auch nach Papinians Ansicht ein breiter Raum für das stuprum übrig. Vor Allem wird ein dauernder Verkehr erfordert worden sein, dann aber auch eine solche Gestaltung desselben, welche ihn gesellschaftsfähig macht. Eine puritanische Auffassung wird freilich in der Offenkundigkeit des ehelosen Zusammenlebens ein besonderes Zeichen von Schamlosigkeit und darum noch eine erhöhte criminelle Qualification erblicken; aber die freidenkende Anschauung der Antike erblickt grade in dem offenen Eingeständniss des Verhältnisses ein ethisches Moment und hat Beziehungen, wie die des Perikles zur Aspasia ebenso zu dulden gewusst, wie sie die des älteren Cato zu seiner nächtlichen Besucherin ${ }^{2}$ ) deutlich missbilligt. Das honore pleno diligere ist also hier gerade das charakteristische Moment, welches das Zusammenleben des Cassianus und der Rufina, wenn nicht legitimirt, so doch legalisirt; indem der Senator seine Geliebte gleich einer Hausfrau hielt, entrückte er das Verhältniss der Sphäre des Verbotenen um so mehr, als die Dame möglicher- und sogar wahrscheinlicherweise gesellschaftlich recipirt worden war; wir haben hier die merkwürdige Erscheinung einer sich selbst von den freilich unvernünftigen Bestimmungen des Gesetzes dispensirenden Gesellschaft vor uns.

Noch ist die Frage aufzuwerfen, worin denn das Disstinctiv dieses Verhältnisses gegen die Ehe lag, da bekanntlich zu dieser ein solenner Contractsabschluss nicht gehörte. Hiemit berührt man freilich einen sehr schwierigen Punct; wir mit unsrer seit Jahrhunderten an die solennisirte Eheschliessung gewöhnten Betrachtungsweise vermögen uns gar nicht in eine Gesellschaft hineinzuversetzen, in der eine so fundamentale Rechtsthatsache wie die Ehe nicht jeweils auf

1) Vgl. Dirksen, civ. Abh. 1, 208; Wlassak, Krit. Studien 114. 2) Plutarch, Cato maior c. 24. 
documentarischer Basis ruhte. Und doch hat es diesen Zustand auch im canonischen Recht des Mittelalters Jahrhunderte lang gegeben und gibt es noch heute nach dem californischen Gesetz und andern nordamerikanischen Gesetzen. Dass damit eine gewisse Rechtsunsicherheit gegeben ist, leuchtet ein; aber man darf sich die Dinge praktisch nicht unlösbar denken. Zunächst ist klar, dass man im $Z_{w}$ eifel bei fortgesetztem Zusammenleben lieber Ehe angenommen haben wird als das Gegentheil, und das findet sich fast mit der Kraft einer Präsumtion ausgesprochen bei Modestin D. 23, 2, 24: 'In liberae mulieris consuetudine non concubinatus sed nuptiae intelligendae sunt, si non corpore quaestum fecit', wodurch desselben Verfassers Bemerkung in D. $48,5,35$ pr. wesentlich begrenzt wird. Doch gilt dies nur, sofern nicht andere Umstände eingreifen; diese können bald für Concubinat, bald mit beweisender Kraft für die Ehe sprechen. Die leitenden Gesichtspuncte deutet Papinian selbst mit grosser Klarheit an; D. 39, 5, 31 pr.: 'An autem maritalis honor et affectio pridem praecesserit, personis comparatis, vitae coniunctione considerata perpendendum esse respondi'. Daran liegt sehr viel; es kann schon die Vergleichung der Personen ein Indiz bilden und man wird die Verbindung des Senators mit einer humilis persona ebenso ungern für eine Ehe nehmen, als man dies beim Zusammenleben zweier social gleich tief Gestellten leicht thun wird; aber freilich ist dies wie jedes Indiz nur in Gesammtheit aller Umstände zu würdigen und zu diesen gehört vor Allem die äussere Ausgestaltung des Verhältnisses, sowohl nach seinem Inhalt als nach seiner Begründungsform. Ein intermittirendes Zusammensein, das in Wahrheit auf ein beständiges Kommen und Gehn hinausläuft - man denke an Kellers grünen Heinrich und Judith ${ }^{1}$ ) - wird anders betrachtet werden als ein dauerndes; aber auch bei diesem kann die Eheabsicht dadurch ausgeschlossen erscheinen, dass die nach dem Stande der Betheiligten zu erwartende feierliche Eheschliessung fehlt. Denn auch das liegt in Papinians

1) Ich meine das Verhältniss, wie es zum Schluss der Erzählung im 16. Capitel des vierten Bandes geschildert wird. 
Worten und sein Zusatz 'neque enim tabulas facere matrimonium' ist sicher nicht dahin zu verstehn, dass die Formlosigkeit des Zusammenkommens unter allen Umständen ganz unbeachtlich ist, das ist vielmehr in verschiedenen Ständen ganz verschieden zu behandeln. Wenn ich nicht irre, liegt gerade in unserm Fall eines der entscheidenden Momente darin, dass es an der Hochzeitsfeier ganz fehlte; der Mann hatte eben nur in Ehren 'geliebt', aber nicht in Ehren gefreit und das war deswegen von grosser Bedeutung, weil es ein Senator war, der feierliche Hochzeit zu machen pflegt, wenn es ihm Ernst ist - nicht umsonst fügt der mit den Worten so karge Jurist bei, dass es sich um einen vir clarissimus handelte.

Freilich dies ist nicht sein einziges Argument, sondern es trat noch ein zweites hinzu, das in Verbindung mit dem ersten einen ganz unwiderleglichen Beweis herstellte. Und hiemit kommen wir zu dem so viel misshandelten Schlusssatz der Stelle. Cassianus hatte eine Tochter der Rufina, indem er sie als seine Ziehtochter bezeichnete, zusammen mit seiner Enkelin zur Erbin seines Vermögens eingesetzt, während er der Rufina selbst ein Legat auswarf. Und von dieser Tochter fand das kaiserliche Auditorium, dass sie vulgo quaesita sei. Dies hat sehr einfach folgenden $\mathrm{Zu}$ sammenhang. Die Tochter entstammte nicht aus einem vor der Bekanntschaft mit Cassianus begangenen Stuprum der Rufina, sondern war eben in jenem Concubinat geboren ${ }^{1}$ ) und wurde darum von Cassianus als seine natürliche Tochter angesehn; demgemäss bezeichnete er sie, um ihre Einsetzung zu rechtfertigen, als seine Ziehtochter. Gerade dieser Umstand konnte nun im Process von den Gegnern ausgebeutet werden und ist es jedenfalls worden. Die Tendenz war dabei wohl von Seite der Processgegner die, der Rufina die Eigenschaft als Ehefrau, welche sie wahrscheinlich auf Grund ihres offenkundigen Zusammenlebens mit dem Verstorbenen in Anspruch nahm, zu bestreiten und sie so als erwerbsunfähig

1) Das hat schon Ranchinus in Otto's Thesaurus 5 p. 247 richtig aufgefasst, aber er steht, soviel ich sehe, mit seiner Meinung ganz allein. Uebrigens hat er diesen Gedanken nicht weiter verfolgt und darum die befriedigende Erklärung des Zusammenhangs nicht gegeben. 
hinzustellen. Die Argumentation wird diese gewesen sein: wäre Rufina Ehefrau, so hätte der Testator das gemeinsame Kind nicht als Alumna, sondern als 'filia' bezeichnet; indem er letzteres thut, legt er ein Zeugniss für den ehefremden Charakter des Verhältnisses ab. Und dieses Argument war in der That ein überaus kräftiges; eben deswegen wird es von Papinian der Entscheidung, offenbar zu ihrer vollgültigen Begründung, hinzugefügt und hat jedenfalls auch unter den kaiserlichen Entscheidungsgründen figurirt. Nicht umsonst sagt der Jurist 'vulgo quaesitam a p paruit'. Es ist durchaus sprachwidrig, hierin eine blosse Relation über ein Thatbestandsmoment zu sehen, wie es die bisherigen Ausleger thun; das 'apparet' deutet auf eine Meinungsäusserung des Obergerichts selbst. Die Unehelichkeit ${ }^{1}$ ) der Tochter wurde nicht als feststehende Thatsache - etwa weil sie aus einem früheren Stuprum der Rufina stammte - der Entscheidung zu Grunde gelegt, sondern war eben unter den Parteien mitbestritten und wurde als Präjudicialpunct erst jetzt festgestellt.

Es ist nicht uninteressant, hier ein Stück des von Scialoja nach Botti's Lesung, leider bisher nur theilweise, veröffentlichten ${ }^{1}$ ) Papyrus Cattaui zu vergleichen; derselbe enthält auf dem Recto eine Anzahl von Gerichtsverhandlungen aus der Kaiserzeit. Da heisst es an einer Stelle, vom 10. Payni des Jahres 115:

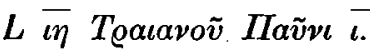

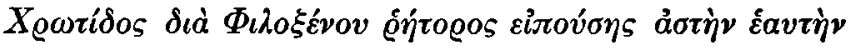

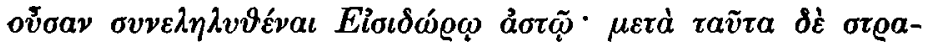

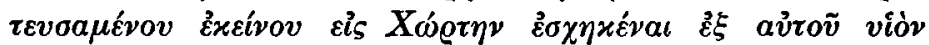

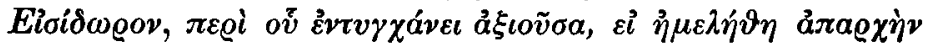

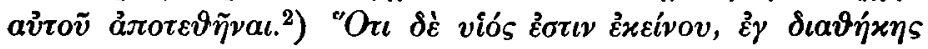

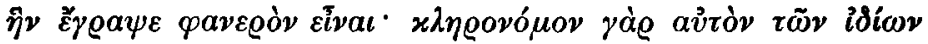

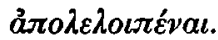

1) Dass auch das Concubinenkind als vulgo quaesitus bezeichnet werden kann, scheint mir zweifellos, wenigstens nimmt Fastus v. Nothum (p. 174 Müller) keinen Anstand, solche Kinder als spurii zu bezeichnen.

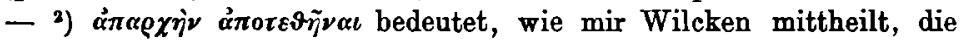
Befreiung von der Erbschaftssteuer, welche für Isidoros als den Sohn des Erblassers in Anspruch genommen wird. 
Hieraus sehen wir nämlich, dass man sich, um die Vaterschaft an einem Kinde zu beweisen, nöthigenfalls auch darauf berief, dass dasselbe von dem behaupteten Vater als Erbe eingesetzt worden sei, und in der That ist dies ein ziemlich schwerwiegendes Argument, welches auch vom Gericht nicht bestritten, sondern nur als gleichgültig bezeichnet wird; gleichgültig im gegebenen Fall deswegen, weil für das Kind eheliche Abstammung in Anspruch genommen wurde, während der Vater als Soldat nicht heiraten konnte. Der Fall liegt dem unsrigen nicht ganz analog; aber er ist ein Seitenstück dazu insofern, als wir in beiden Fällen den Indizienbeweis aus dem Testament über die Frage der Vaterschaft resp. Ehelichkeit angerufen sehen.

Demnach ist der Gedankengang der Digestenstelle folgender: Die Kaiser haben entschieden, Rufina ist nicht Ehefrau; denn es liegt zwar ungescheutes und gesellschaftlich anerkanntes Zusammenleben vor, aber dieses ohne Ehecontract kann für Ehe um so weniger angesehen werden, als die Tochter aus diesem Umgang vom Vater nur als Ziehtochter im Testament eingesetzt ist. Aber anderseits ist solcher Concubinat kein Stuprum, und daher tritt auch keine Incapacität ein. Die nur indirect ausgesprochene Voraussetzung dabei ist, dass die Concubine als solche nicht zu den erbunfähigen mulieres probrosae zu zählen ist. Dies ist aber auch sonst zweifellos, da Vermächtnisse an Concubinen wiederholt in den Digesten als giltig behandelt werden. ${ }^{1}$ ) Wie man dies mit dem bei ihnen immerhin vorhandenen Caelibat vereinbart hat, steht dahin; die Thatsache ist ausser Zweifel.

1) z. B. $34,1,15 ; 34,2,35 ; 32,29$ pr. und 41,$5 ; 33,2,24,1$. In D. 34, 9, 14 liegt kein Concubinat vor, hier ist die Entscheidung eine andere. - Wenn Marcellus D. 23, 2, 41, 1 der Concubine des 'non patronus' die Matronenqualität abspricht, so erklärt er sie doch nicht für eine mulier probrosa; sie kann nur nicht wegen Adulterium angeklagt werden (D. 48, 5, 14 pr.), weil das nur bei Matronen (und dazu zählt auch die concubina liberta) möglich ist. 